\title{
TNT: A Tactical Network Test platform to evaluate military systems over ever-changing scenarios
}

\author{
Paulo H. L. Rettore, Johannes F. Loevenich, Roberto Rigolin F. Lopes and Peter Sevenich
}

\begin{abstract}
This paper addresses the challenge of testing military systems and applications over different communication scenarios with both network conditions and user data flows changing independently. We assume that systems developed to handle ever-changing communication scenarios are more likely to be reliable and robust during real military operations. Therefore, we propose the Tactical Network Test (TNT) platform to automate the evaluation of military systems and applications over real military radios using a reproducible test methodology. TNT has four main goals (i) the creation of QoS-constrained data flows; (ii) the execution of models to change network conditions; (iii) a performance evaluation of a military system over ever-changing conditions; and (iv) the monitoring and performing data analysis. Our platform was used to execute experiments in a VHF network by sending uniformly distributed data flows during seven different communication scenarios, either generated by a stochastic model or mobility models. The experimental results are used to discuss the military system's performance by quantitative analysis using network metrics and the test scenario characterization through mobility metrics.
\end{abstract}

Keywords-Testing Tactical Systems, Military Systems, EverChanging Network Scenario, Mobility Models, Tactical Networks

\section{INTRODUCTION}

Military communication systems deployed at the edge of tactical networks must be robust to the frequent topology changes in Mobile ad-hoc Networks (MANETs) [1], [2]. Therefore, military systems use multi-layer control mechanisms to change its configuration using feedback from different network devices, such as radios, routers and controllers. Moreover, tactical networks are characterized by the low bandwidth, high delay, and frequent link disruptions, pushing military systems to deal with challenging network conditions. Tactical networks support military operations providing the means for network-centric warfare, among military units in large areas, through heterogeneous networks combining different communication technologies, such as High Frequency (HF), Ultra High Frequency (UHF), Very High Frequency (VHF) and Satellite Communications (SatCom). The Command and Control (C2) services supporting military units have a wide range of Quality of Service (QoS) requirements, including priority, reliability, security, time of expire and so on. This

Manuscript received March 02, 2021; revised xxx xx, 2021.

P. H. Rettore, J. F. Loevenich, P. Sevenich and R. R. F. Lopes are with the Communication Systems Department (KOM), Fraunhofer FKIE, Bonn, 53177 Germany, e-mail: \{paulo.lopes.rettore, johannes.loevenich, roberto.lopes, peter.sevenich\}@fkie.fraunhofer.de.

J. F. Loevenich is also with the Institute of Computer Science 4, University of Bonn, Bonn, Germany e-mail: s6joloev@uni-bonn.de fact motivated us to develop a test platform to verify if the tactical systems can meet QoS requirements over everchanging communication scenarios.

In this investigation, we introduce the Tactical Network Test (TNT) platform to test military systems over communication scenarios with independent changes in both network conditions and user data flows. TNT provides the means of systematically test military systems before using them in the operational field. To the best of our knowledge, recent literature often uses static or periodic changes, reducing the test condition to a specific set of changes/scenarios, making the evaluation of the systems restrict to these contexts. Moreover, most of the literature lacks a test scenario characterization, in terms of mobility, which is essential to comprehend the characteristics of the network entities involved in the test.

The present study is also motivated by the lessons learned in our previous investigations defining models to create everchanging communication scenarios [3]-[8]. The goal is add a variety of reproducible scenarios and to provide a software platform to support the test of tactical systems over real military radios. Therefore, we proposed TNT platform designed to automate the evaluation of military systems (also called middlewares, brokers or proxies) and applications over a variety of user data flows and network conditions. We assume that, systems developed to handle both ever-changing network conditions and ever-changing user data flows are more likely to be reliable and robust during real military operations. In summary, the contributions of this paper are as follows:

- Design of a software platform to automate the test of military systems/applications in tactical networks. The main design decisions are (i) model $\mathfrak{M}_{A}$ to create data flows, (ii) model $\mathfrak{M}_{B}$ to create network conditions, (iii) monitoring and data analysis;

- Introduction of two models to transform sequences of network states in a mobility pattern and vice-versa.

- A mechanism to change the military radio modulation following mobility traces, and to create link disconnections using a relay and controller in radios with wired antennas;

- An adaptive mechanism to shape the user data flows to the network conditions, therefore, mitigating buffer over-flow in military radios.

The rest of this paper is organized as follows, Section II discusses recent investigations also proposing test environments for military systems in tactical networks. Section III explains the design of TNT platform, highlighting the models and the methodology to create ever-changing communication scenarios in a real test-bed. Section IV compares experimental results using patterns of link data rate change generated with 
stochastic models and mobility models. Section V discusses the lessons learned and the current limitations of our test platform. Lastly, Section VI concludes the paper and highlights future improvements.

\section{RELATED WORKS}

This section discusses our previous investigations introducing models to create ever-changing communication scenarios over real military radios. Together with the recent literature also introducing test platforms with similar goals to TNT.

\section{A. Ever-changing communication scenarios}

In previous investigations, we introduced stochastic models to create ever-changing communication scenarios with the user generated data flows and the network conditions changing independently [4], [6]-[9]. These investigations assume that the military system being tested is deployed in the real platform, which is composed by the radio, router, switches and user-facing node. The goal is to design and test the control mechanisms handling the ever-changing communication scenarios. For example, in [5], [8] we introduced multi-layer control mechanisms doing flow control, handling packet loss and mitigating radio buffer overflow. Our stochastic models use Markov chains to create the communication scenarios therefore the results can be replicated for independent verification.

The main question addressed in these investigations is how to guarantee that the models are creating enough variations in the communication scenarios to test the system's ability to handle unpredictable conditions including link disconnections and burst of messages. The investigation in [3] introduces a multi-layer stochastic model to compute the probability of message delivery. The goal is to maximize the probability of message delivery by adding redundancy to the user data flows.

\section{B. Test platforms}

The authors in [10] developed an emulation environment and scenario as a result of a joint effort by the NATO science \& Technology Organization's IST-124 task group. The emulated test-bed, using EMANE, can support different communication technologies such as HF, UHF, VHF and SatCom due to its generic Radio Frequency (RF) propagation model. Moreover, they created a military scenario called Anglova in a fictitious area of Fieldmont with 157 vehicles (network node) over the course of two hours. This scenario is called realistic by the authors because military experts designed the scenario. In [11], the authors proposed a Tactical Network Integration Test Framework for simulation and emulation composed of three test environments, namely simulation, high fidelity emulation, and scalable emulation. They started with a high fidelity testbed as a baseline, in order to build the same scenario for all three environments and test them comparing the performance in order to ensure consistency in the experimentation. Then, they increased the network size and evaluated all environments again comparing against each other.

In [12] the authors proposed an emulated test-bed enabling experimentation of Software-defined Networks (SDN) deployed in tactical scenarios. The emulation can be used to run various applications or traffic generators in diversely configurable SDN setups. The SDN layer can be configured to apply a specific architecture and deployment of SDN nodes. Additionally, the network scenarios change accordingly to the Anglova scenario [10]. The investigation reported in [13] developed a test-bed for Software-defined Tactical Network (SDTN) tests in an emulated environment using MininetWifi emulator. A specific SDN architecture is applied using a distributed controller deployment with a global and multiple local controllers. The network can change using mobility and connectivity patterns that are adjusted to represent conditions matching a required test-case. The user data flows is created by traffic generator tools and their system includes a visualization interface showing the network metrics. In the same direction, iTrinegy commercialize a solution named Software Defined Test Networks [14] to emulate tactical networks enabling reproducible, realistic and dynamic network profiles.

These studies provide emulated/simulated test-beds, some of them focusing on SDN-capabilities, and others have generalpurpose applicability. They ensure network changes using Anglova scenario or mobility patterns and user data flows through traffic generator tools or control messages of routing protocols. However, most of these studies lack a clear characterization of the test methodology defining the application, the military system, and network view, making the reproducibility of them a challenging task. In addition, other investigations [15]-[18] developed emulated test-beds for different purposes but lacking a precise definition of proposed models and the discussion regarding the ever-changing network conditions and the user data flows.

Furthermore, in general, military systems must deal with resource-constrained radio devices in terms of buffer size, modulation, and power-constraints. Many studies [7], [8], [19]-[24] developed solutions such as middlewares, brokers, or proxies to adapt the user data flows to the network constraints.

\section{Remarks}

Changes in the network and user data-flow can significantly affect the overall system performance in aspects such as routing and QoS. However, recent literature lacks the discussion of testing military systems over ever-changing communication scenarios in emulated and real environments. Meaning that most studies use static or periodic changes and don't develop reproducible methodologies, restricting/lacking the evaluation and characterization of such test scenario. The authors in [25] discuss the importance of categorizing network scenarios to improve the description of realistic node mobility in the air. Taking into account mobility metrics, the authors in [26] analyzed a list of available models meeting the requirements of tactical scenarios and also identifying models that can be extended to meet such requirement.

Regarding ever-changing communication scenarios, the present investigation complements our previous researches and advances them in different manners by (i) introducing mobility models to create network scenarios that can be characterized by mobility metrics; (ii) proposing a mobility model based 


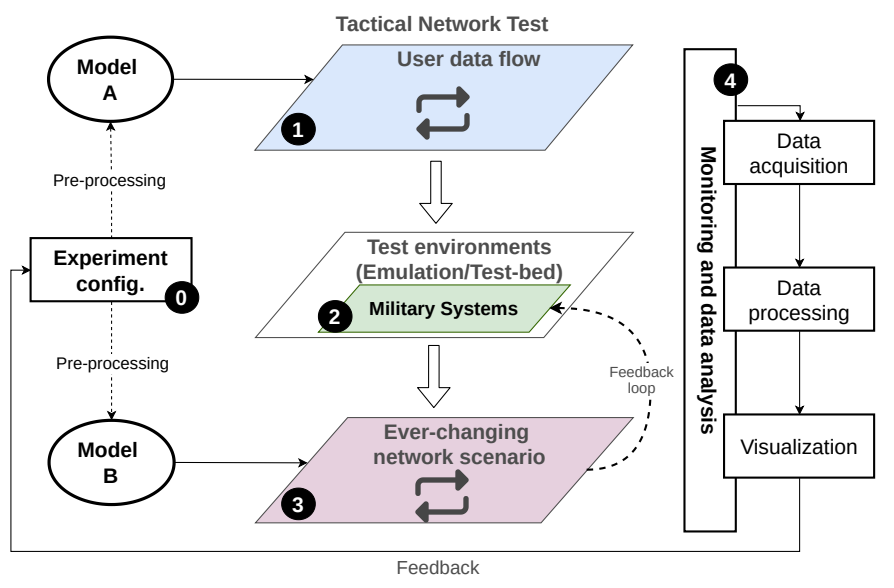

Fig. 1: Design of TNT platform.

on the Markov chain; (iii) modeling the communication area in order to convert any sequence of network states (link data rate) to a mobility trace and vice-versa; (iv) proposing a general model for user data flow changes; and finally, (v) wrapping these methods together with a monitoring and data analysis approach, providing a software platform to automate performance tests in tactical networks.

Different from the literature on test platforms, we designed a general-purpose platform independent of SDN concepts. Besides that, TNT is designed decoupling the test of military systems in three layers, namely the application, the system itself, and the network layer. Therefore, it uses the model $\left(\mathfrak{M}_{A}\right)$ to generate a wide range of QoS-constrained user data flows at the application layer. On the network layer, TNT proposes models $\left(\mathfrak{M}_{B 1}\right.$ and $\left.\mathfrak{M}_{B 2}\right)$ for creating different network scenarios and deploying them in the real tactical network instead of the emulated network. We also designed a prototype to create link disconnection in real stationary radios.

From the military system perspective, specifically, the mechanisms to adapt the user data flow to the network constraints, most of the studies mentioned above require a stack of software to work properly and these systems need to be replicated each hop, making the scalability a tough task. On the other hand, TNT proposes an adaptive shaping mechanism using Linux queue discipline and radio feedback, such as data rate and buffer usage, to adapt the user data flow to the network constraints. Compared to the literature solutions, we argue that our adaptive shaping mechanism is a low-cost and easy-to-use approach handling resource-constrained radio devices.

\section{The TNT Design}

In this section, we describe the Tactical Network Test (TNT) platform designed to automate tests to evaluate military systems and applications over a variety of network conditions and user data flows. The test results are composed by the system performance evaluation and the test scenario characterization. The TNT design is composed of three main components controlled and monitored by the TNT platform during an experiment, namely the user data flows, the military system being tested and the network conditions, as illustrated in Fig. 1. These are TNT's building blocks to define the means to configure an experiment, to execute the experiment over a real platform with at least two nodes, and to analyze the experimental results. In this figure, these three steps are defined as follows:

(0) Experiment configuration: is composed of two models defining the user data flows (1) and the network conditions (3);

(2) Military systems: in this phase TNT deploys a middleware, broker, or proxy at the nodes in the test-bed. This is the system subject of the performance tests executed by TNT;

(4) Monitoring and data analysis: TNT collects the pertinent logs and process them for quantitative analysis of the experimental conditions and the system performance.

In the application layer (1), the user data flows are generated by a model that creates different patterns of data flows. From the network perspective (3), TNT creates an infrastructure that uses patterns of data rates change and mobility traces, simulating a wide range of network scenarios. These scenarios are deployed in a real test-bed or emulated environments in order to test a given military system (2). In this investigation, TNT is instantiated to deploy network scenarios in a test-bed with real military radios. The next sections discuss in details the main building blocks of the TNT platform.

\section{A. Experiment configuration}

The first step is to configure the experiment by using the two models $\mathfrak{M}_{A}$ defining the user data flows, and $\mathfrak{M}_{B}$ defining the network conditions and the test environment. This test environment can be either created by an emulation software or a real military test-bed like in our laboratory. At the application layer, TNT defines a model to create data flows $\mathfrak{M}_{A}$, allowing the users to input parameters such as number of messages, size and inter-message delays to define different patterns of data flows. Moreover, TNT uses model $\mathfrak{M}_{B}$ to configure the network setup by defining how the radio link data rate will change during the experiments. This is done by selecting and providing one of the three input types, namely (i) a sequence of link data rates; (ii) a probability matrix (e.g. a Markov chain) for the corresponding network states; or (iii) a mobility trace $(x, y$, time $)$ created from mobility models. Finally, TNT defines the network topology by setting the number of nodes and the radio links connecting them. The latter is defined by parameters defining the communication technology, which has a range (kilometers), set of modulations defining nominal link data rates and the overall end-to-end delay.

\section{B. Creating user data flows}

In order to introduce the element of chance at the application layer, TNT propose a model to create user data flows that can simulate different applications through the generation of different traffic flows. For this purpose, we extend the user data flow model defined in our previous investigation [4] to a model 


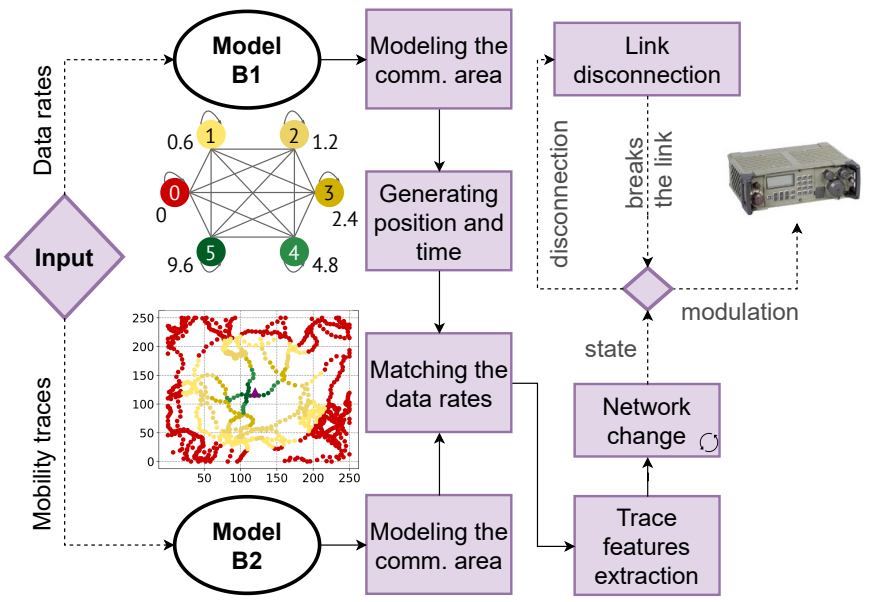

Fig. 2: Design of ever-changing network scenario.

$\mathfrak{M}_{A}$, also representing the variations of the user data flows created by different sequences of messages (modeled as a set of IP packets), message sizes, and sending rate. Formally, $\mathfrak{M}_{A}$ extends the data flow model by the quadruple $\langle\eta, \theta, \omega, \mu\rangle$, where $\eta$ defines the number of messages, $\theta$ the inter-message delay, $\omega$ the message size, and $\mu=\langle\rho, \mathfrak{R}, T o E, \cdots\rangle$ again represents a $m$-tuple that brings $m$ different QoS metrics such as $\rho$ representing the message priority, $\mathfrak{R}$ addressing its reliability, $T_{O} E$ the time of expire and so on. In addition, $\eta, \theta$ and $\omega$ are defined as functions sampling the number of messages, the inter-message delay and the message size using a probability distribution chosen by the user. This representation increases the diversity of the outputs created by TNT and as a result experiments can be conducted with more realistic user data flows instead of scenarios with static/periodic changes or specific applications. Meaning that the system performance will reflect these specific test conditions, and no further assumption can be supported.

\section{Creating ever-changing network scenarios}

To create ever-changing network scenarios TNT introduces two models $\mathfrak{M}_{B 1}\left(\Sigma, S, \lambda, C, f, n_{\text {ref }}\right)$ and Model $\mathfrak{M}_{B 2}\left(\Gamma, C, n_{\text {ref }}\right)$ transforming sequences of data rates into mobility patterns and vice-versa, respectively. Both models also define parameters to configure the test environment, meaning that they can be used to initiate the changes of the network states through a radio interface and a link disconnection prototype. The input parameters for model $\mathfrak{M}_{B 1}$ are as follows: $\Sigma=\left(\sigma_{1}, \ldots, \sigma_{n}\right)$ represents a sequence of data rate changes over system state $S=\left\{s_{0}, s_{1}, s_{2}, s_{3}, s_{4}, s_{5}\right\}$, which can be isomorphically mapped to the set of data rates $\{0.6,1.2,2.4$, $4.8,9.6\}$ kbps supported by the radios. It should be noted that the system state $S$ can be defined in any desired way to match with the modulation of the radios used in the test environment.

Moreover, the sequence of states $\Sigma$ can either be sampled using probability distributions, such as used in [4], [7], [8], or manually defined by the user. The input parameter $\lambda$ defines when the movement starts $s_{s t}$, and the time distribution for state updates $s_{t i}$, meaning that the model changes or disconnects the network link in the test environment according to the time distribution defined by $\lambda$. Next, the parameter $C$ represents the communication area with respect to the link quality defined by the current data rate $\sigma_{i} \in \Sigma$. Moreover, different distributions can be applied to sample the node positions over space using the probability density function $f$.

In the following, we will explain one exemplary approach to generate this kind of communication area by using circular areas expanding around a reference node $n_{r e f}(x, y) \in$ $\mathbb{R} \times \mathbb{R}$. Using the communication area $C$ and inverse transform sampling, $\mathfrak{M}_{B 1}$ outputs an enriched mobility trace $\Gamma_{E}=\left\{\left(s_{t i}, p_{1}, \sigma_{1}\right), \ldots,\left(s_{t i}, p_{N}, \sigma_{n}\right)\right\}$ composed by points $p_{1}, \ldots, p_{n}$ in a bi-dimensional vector space $V \times V$ distributed over time $s_{t i}$, matching the correspondent $\sigma_{i} \in \Sigma$. In contrast, $\mathfrak{M}_{B 2}$ is used to transform a mobility trace $\Gamma=$ $\left\{\left(\lambda_{1}, p_{1}\right), \ldots,\left(\lambda_{n}, p_{n}\right)\right\}$ into an enriched mobility trace $\Gamma_{E}=$ $\left\{\left(s_{t i}, p_{1}, \sigma_{1}\right), \ldots,\left(s_{t i}, p_{N}, \sigma_{n}\right)\right\}$ containing the respective data rate $\sigma_{i}$. In other words, $\mathfrak{M}_{B 2}$ focuses on the transformation function, mapping the $p_{1}, \ldots, p_{n} \in \Gamma$ to the ring-shaped areas $C$ with respect to a reference node $n_{r e f}(x, y) \in \mathbb{R} \times \mathbb{R}$, describing a specific link data rate.

These models aim to enrich the discussion around the test scenario by extracting mobility features that describe military unities in a field such as humans, ground vehicles, aerial vehicles, or unmanned vehicles. In this investigation, the military radios support five different modulations which are represented as a Finite State Machine with six states $(S)$, where state $\{0\}$ represents disconnection, states $\{1,2,3,4$, $5\}$ correspond to the radio data rates of $\{0.6,1.2,2.4,4.8$, $9.6 \mathrm{kbps}$ as shown in Fig. 2. It is important to notice that TNT assumes that the radios have an interface (e.g. Simple Network Monitor Protocol (SNMP)) to change the link data rates (radio modulation) or adding an attenuation (e.g. using a channel emulator) to force the radio to change its modulation.

1) Model $\mathfrak{M}_{B 1}$ : Transforming sequences of data rates into mobility patterns: Model $\mathfrak{M}_{B 1}$ creates mobility patterns from a given sequence of data rates $(\Sigma)$, resulting in an enriched trace $\Gamma_{E}=\left\{\left(s_{t i}, p_{1}, \sigma_{1}\right), \ldots,\left(s_{t i}, p_{N}, \sigma_{n}\right)\right\}$, that describes a given mobility pattern. In this sense, we describe the required steps to transform a sequence of data rates in a mobility trace. First, we define a mapping of the system system state $S$ to a set $A_{1}, \ldots, A_{n}$ of circular areas inspired by the wave propagation of omni-directional antennas. After that, we use inverse transform sampling to create the positions $p_{1}, \ldots, p_{n}$ and the resulting enriched mobility trace $\Gamma_{E}$. Besides, the mobility statistics from $\Gamma_{E}$ are extracted and the resulted trace, with the network states, is used to change the radio link data rate or create link disconnections. These steps are discussed in detail as follows.

\section{Modeling the communication area:}

Let us define the area of communication $C=A_{1} \cup \cdots \cup A_{n}$ for a node with $|S|$ different system states as the composition of $|S|$ nested circular areas. The radius $r_{i}$ of the circular areas is defined through the system state $s_{i}$ of the corresponding node. As a result, we can describe the area for a given number of different system states $S$ by slicing the circle 
for the maximum radius (system state), thus $n=|S|$ rings of communication. The ring boundaries is defined as inner boundary $R_{\text {inner }}$ and the outer boundary $R_{\text {outer }}$. In this investigation, we assume that both boundaries have a magnitude $M$ in meters, as defined in (1), allowing create different ranges of communication. The test cases in this paper consider an environment area of 25 square kilometers and $M=2 \mathrm{~km}$, therefore, modeling the node communication area with a radius of $20 \mathrm{~km}$ and the disconnection is defined as the area beyond this radius. Notice that, the communication range depends on radio technology, and here we are inspired by our VHF radios which have this communication capability.

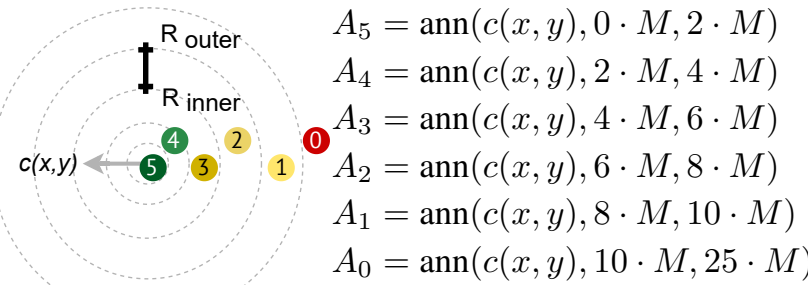

As a result from the previous observations, each system state $s_{i} \in S$ can be mapped to a ring-shaped area $\left.A_{i}=\operatorname{ann}\left(c(x, y), R_{\text {inner }}, R_{\text {outer }}\right)\right)$ with the node center position $c(x, y)$. The resulting communication area $C=A_{1} \cup \cdots \cup A_{n}$ defines the sample space to create the points $p_{1}, \ldots, p_{n}$ of the raw mobility trace $\Gamma_{R} \subset \Gamma_{E}$ using the probability density function $f$. Note, that this specific representation for the communication area is inspired by the wave propagation of omnidirectional antennas like the radios used in our laboratory. But, it can easily be replaced by any another representation by defining a mapping from the system state $S$ to any arbitrary set $A_{1}, \ldots, A_{n}$ defining the communication area $C$.

Generating position and time: Given a specific node with position $c(x, y)$, TNT implements inverse transformation sampling to create the points $p_{1}\left(x_{1}, y_{1}\right), \ldots, p_{n}\left(x_{n}, y_{n}\right)$ of the raw mobility trace $\Gamma_{R}$, sampled from the circular areas $A$ generated in the last step. For this purpose, let us assume that we have a reference node, which can be a base station or a neighboring node sharing its location, with position $n_{\text {ref }}(x, y)$. Depending on the link quality (system state) of the node with position $c(x, y)$ and the reference node $n_{r e f}(x, y)$, TNT computes the inner radius $R_{\text {inner }}$ and outer radius $R_{\text {outer }}$ of the annulus $A_{i}$, as shown in (1). In simple terms, this annulus describes the space of points matching the communication areas (say link data rates) of the two nodes with their distance $\left|c(x, y)-n_{r e f}(x, y)\right|$. Inverse transform sampling now enables TNT to sample a point from this communication areas depending on the link quality between the two nodes.

To this end, let $X$ be a random variable describing the distance $r$ of a point $p(x, y)$ to the center $c(x, y)$ (current node position). Then, the Probability Density Function (PDF) describing the relative likelihood of the distance of a point to the center $c(x, y)$ is shown in Eq. (2). Furthermore, in Eq. (3), using the PDF, we get the value of the Cumulative Density Function $(\mathrm{CDF})$ for distance $d$ by calculating the integral of $f(r)$ from $R_{\text {inner }}$ to $d$.

$$
\begin{gathered}
f(r)=\frac{2 r}{R_{\text {outer }}^{2}-R_{\text {inner }}^{2}} \\
F(X=d)=\int_{R_{\text {inner }}}^{d} f(r) d r=\frac{d^{2}-R_{\text {inner }}^{2}}{R_{\text {outer }}^{2}-R_{\text {inner }}^{2}}
\end{gathered}
$$

Moreover, the inverse of $F(X=d)$ in $d$ is:

$$
d=\sqrt{u\left(R_{\text {outer }}^{2}-R_{\text {inner }}^{2}\right)+R_{\text {inner }}^{2}}
$$

Now, TNT can use inverse transform sampling, Eq. (4), to generate values of $X$ which are distributed according to $F$. This works as follows: (1) generating a random number $u_{1}$ from the standard uniform distribution in the interval $[0,1]$; (2) finding the inverse of the desired CDF $F^{-1}(d)$ and (3) computing $X=F^{-1}(u)$.

Once TNT sampled the distance $X=d$, it can compute the $x$ and $y$ coordinates of the point $p(x, y)$ as shown in Eq. (5) by first sampling the corresponding angle $\alpha$ with respect to an uniformly distributed random number $u_{2}$ and calculating $x$ and $y$ using $\alpha$ afterwards. As a result, it generates a sequence of positions that we call Raw trace $\Gamma_{R}$. Moreover, TNT varies this model by restricting the coordinates distribution to the nearest position using the euclidean distance, thus generating a trace of $n$ new samples as shown in Eq. (6). This approach allows the creation of new traces, named Shortest trace, restricting the node movements to the shortest distance using the same uniform coordinates distribution.

$$
\begin{aligned}
& \alpha=2 \cdot \pi \cdot u_{2} \\
& x=d \cdot \cos \alpha \\
& y=d \cdot \sin \alpha \\
& \text { distance }=\text { euclidean }\left([x, y],\left[x_{-1}, y_{-1}\right]\right) \\
& {[x, y]= \begin{cases}{[x, y]} & \text { if } \min (\text { distance }) \\
{[E q .5,6]_{1 \ldots n}} & \text { otherwise }\end{cases} }
\end{aligned}
$$

In the last step, the model outputs a trace file with node positions and link data rate (node state) at a given time, node $_{i}=\left[x, y\right.$, time $\left._{\text {state }}\right]$. In this sense, to create positions over time TNT defines when the node starts to move $s_{s t}$ and how long the node will stay at the current state before moving to the next state $s_{t i}$. For example, $s_{s t}=0$ means that the node starts moving from the beginning of the experiment and, on the other hand, $s_{s t}=60$ indicates that the movement will starts after 60 seconds.

To create a new variation of the Raw trace $\Gamma_{R}$, TNT uses linear interpolation to connect the points in space-time. This process outputs a new trace file, named Filled trace $\Gamma_{E}$, with smooth movements. Then, for each node position and time node $_{i}=[x, y$, time $]$ and node $_{i}+1=[x, y$, time $]$, TNT interpolates $n_{p}$ new positions as shown in Eq. (7). The parameters used to generate the sequence of states as well as the stochastic model used as input to this model will be discussed later in Section IV together with the results and statistics of the experiments. It should be noted, that $s_{t i}$ 
equals $\lambda_{i}$ for $\Gamma_{R}$, but not for the Filled trace defined by $\Gamma_{E}$. Furthermore, we cannot label these new node positions replicating the last state or interpolating them, due to the state changes is based on the communication area model, as discussed in Modeling the communication area, thus requiring one more step called Matching data rates described next.

$$
\begin{gathered}
p_{x}=x_{i}+\left(x_{i+1}-x_{i}\right) / n_{p} \\
p_{y}=y_{i}+\left(y_{i+1}-y_{i}\right) / n_{p} \\
p_{t}=t_{i}+\left(t_{i+1}-t_{i}\right) / n_{p}
\end{gathered}
$$

Matching data rates: Here, TNT matches the node position to the respective ring specified previously in (1) and assigns the positions to the correspondent network state. At this point, TNT ensures that any trace must follow the communication area modeled before.

Trace features extraction: With the node's trace node $_{i}=$ $[x, y$, time, state], TNT performs the features extraction in order to describe and characterize the node behavior based on its mobility. It computes metrics like distance from the base station (reference node), travel distance, speed, and acceleration between positions, adding these features to the final trace file. The goal is to enrich the visualization and analysis of test scenarios used in a particular experiment as well as use the trace file to provides the network changes that it is discussed later in this section.

2) Model $\mathfrak{M}_{B 2}$ : Transforming mobility pattern into sequences of data rates: Model $\mathfrak{M}_{B 2}\left(\Gamma, C, n_{r e f}\right)$ transforms mobility patterns into network states and it is used to change the radio modulation or cause link disconnections during the experiment. Therefore, TNT can match mobility patterns with patterns of data-rate change and execute it over real tactical radios. Thus, to transform mobility patterns into data rates, TNT can use any tool that implements mobility models and export a trace file, such as MobiSim [27] and BonnMotion [28]. The tests discussed later in this paper uses BonnMotion due to its flexibility and also implementing several mobility models like Random Waypoint (RWP), Random Walk (RW), Probabilistic Random Walk (PRW), Gauss-Markov (GM), Manhattan Grid (MG), Disaster Area (DA), and so on. This tool creates and analyzes mobility scenarios that allow us to investigate the characteristics of such MANET' scenarios and test them over real tactical network with TNT orchestrating the test experiment.

First, BonnMotion is used to define the mobility model and its parameters to create network scenarios. Then, after executing the trace generator and exporting the correspondent trace file $\Gamma$, the TNT parses this file in order to adapt it to starts the matching process by the definition of a communication area $C$ and a node reference $n_{r e f}$, as discussed before. Differently from model $\mathfrak{M}_{B 1}$, model $\mathfrak{M}_{B 2}$ does not need the Generating position and time for obvious reasons. However, the following steps are still required: Modeling the communication area, Matching data rates, and Trace features extraction processes discussed in Section III-C1. Later, in Section IV, we demonstrate a set of mobility models we choose to create different network scenarios, and we applied them in our real test-bed.

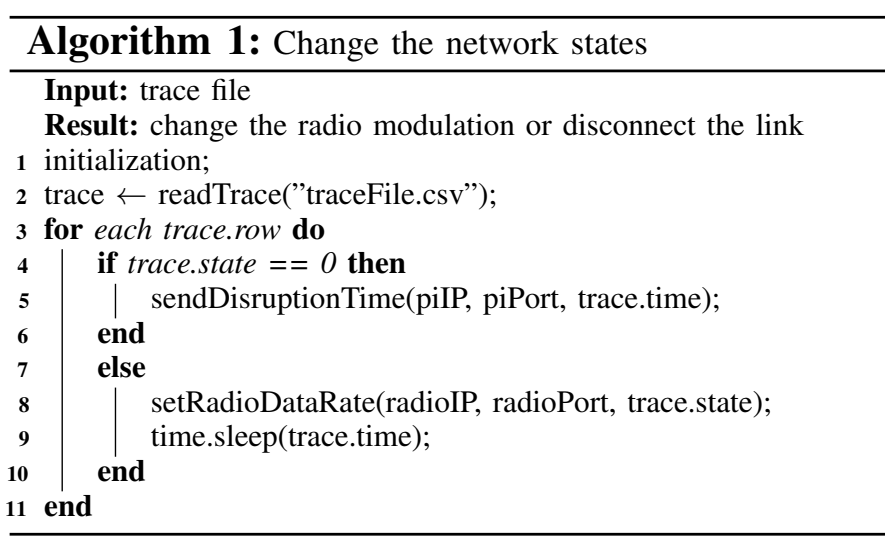

3) Network change: To create network changes based on the mobility traces, TNT uses the radio's SNMP interface to change the nominal link data rates and the link disconnection prototype to create disruptions. Since our test-bed is using radios with wire antennas inside a laboratory, it is not possible to create disconnections moving node like in emulation platforms that we can simulate the node moving away from the communication range. Notice that, real experiments could be done in special military facilities, however, these experiments are costly and can limit the range of tests compared to what we can do simulating such behavior in the laboratory. Therefore, TNT proposes a low-cost and easy-touse link disconnection prototype designed with a controller (Raspberry-Pi) and a coaxial relay. This device creates the link disconnection between nodes (state $\{0\}$ in Model $\mathfrak{M}_{B 1}$ ). Notice that, TNT could also connect to a channel emulator creating link disconnections if a proper interface is developed.

The changes on the network are done by using the mobility trace as input and two different interfaces depending on the node state (i) connected: the interface with the radio (to change its modulation) and (ii) disconnected: the interface with the link disconnection prototype (to 'cut' the wired antenna using a relay). If the network state is $\{0\}$, the script sends a message to the controller to disconnect the link. Otherwise, TNT uses the SNMP interface to set the radio link data rate. Algorithm 1 shows the pseudo-code describing these actions. After the necessary initialization in line 1 , the trace file is read in line 2 and the node time-series and its correspondent states are extracted. Then, the time interval between observations is computed and saved (trace). For each node observation (line 3) a condition (lines 4 and 7) determines which procedure will be performed next. If the node state is 0 , then a socket is created to connect to the disconnection controller using the IP address and the port number (line 5). After the link disconnection, the client receives data from the server unlocking the client to go to the next network state. If the node state is not $\{0\}$, then, the radio modulation will be changed (line 8). The function to set the radio data rate uses as parameters the radio IP, radio port, and the network state (trace.state). Finally, in line 9, the client sleeps for the given time window (trace.time) corresponding to the node mobility and the process starts again to set the next network state or to finish the test. 


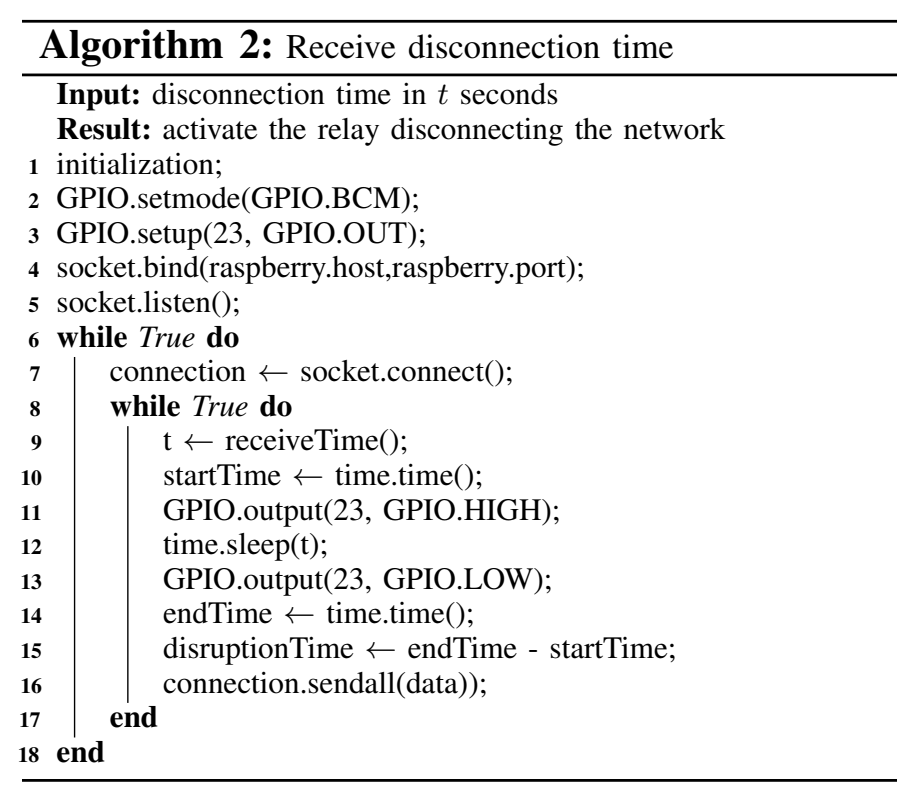

Link disconnection: The link disconnection prototype consists of a relay connected to the coaxial cable between the radios. Moreover, the relay needs $12 \mathrm{v}$ to work, requiring a step-up device converting $5 \mathrm{v}$ to $12 \mathrm{v}$. In addition, there is a circuit with a transistor and resistor to open/close the electric pulse and activate the converter module. TNT also uses an attenuator in order to increase the signal resistance provided by the radio antenna and making the relay works properly. Finally, the Raspberry-Pi acts as a controller to deactivate/activate the relay in a specified time interval, causing connection/disconnection, respectively. The code describing how to build this prototype is available on Github ${ }^{1}$. Algorithm 2 explains the program implemented on the server-side to listen to disconnection requests.

After the initialization in line 1, lines 2-3 define the motherboard model and the $23^{r d}$ General Purpose Input/Output (GPIO) pin is set for output. In Lines 4 and 5, a socket is bound to the Raspberry port and listens for connection requests from the client. If there has been a request (line 6), a connection is established in line 7. If the connection establishment was successful (line 8), then the time interval $t$ is received from the client (trace.time) in line 9. The current time is saved as the start time of the disconnection (startTime) in line 10. GPIO is set high to start the relay, which causes the cable disconnection (line 11). The $16^{\text {th }}$ pin port in the assembly architecture is GPIO23. The disconnection is continued in line 12 for the specified time interval $(t)$. GPIO is set low to stop the relay, which causes the connection re-establishment in line 13. The current time marks the end of the disconnection time (endTime) in line 14. The time interval of the disconnection is calculated in line 15 (endTime - startTime) and this information is sent back to the client in line 16 .

\footnotetext{
${ }^{1}$ https://github.com/prettore/link-disconnection-prototype.git
}

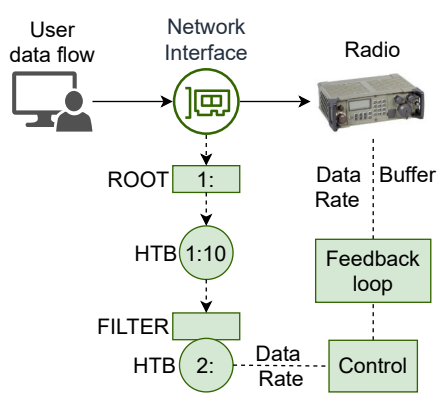

Fig. 3: Design of a queuing discipline for military system.

\section{Military systems}

After introducing TNT's mechanisms to create everchanging user data flow and network conditions, the next step is to design a military system as shown in Fig. 1 (2) in order to test it over different scenarios. Military systems have to manage the limitations of the radio devices, such as link data rate and buffer size. We noticed that the limited buffer size combined with the ever-changing network scenario and user data flow (say message size and time distribution) results in a buffer overflow [29]. This fact imposes a restriction on the range of experiments TNT could support. Therefore, we created a mechanism that can handle the radio buffer overflow by dynamically shaping the data flow, controlling the buffer usage. Such a mechanism is considered here a military system supported by the TNT, but any other system could be deployed on the network to be tested as well. The adaptive shaping mechanism works using Linux Queueing Discipline (QDISC) and information gathering from the radios, such as data rate and buffer usage through SNMP. Fig. 3 shows the design of the hierarchical queues as well as the control mechanism to change the queue rules based on the data rates (which is an abstraction of mobility for the test-bed) and buffer occupancy reported by the radio.

As mentioned before, the goal here is to avoid buffer overflow in resource-constrained devices. To do so, TNT dynamically uses Traffic Control (TC) to shape the data flow based on the feedback loop which gathers the radio buffer occupancy and data rate. Then, TNT creates two queues, where the first Hierarchical Token Bucket (HTB) queue is responsible to shape the data flow based on the maximum data rate supported by the radios, which is $9.6 \mathrm{kbps}$ in our test-bed. This queue aims to hold bursts of messages based on the radio communication boundary. Next, TNT filters all interested data traffic (sender and receiver IP address, UDP packets only, and even the application port), restricting the queues to have only the desired traffic. Notice that, these filters can be changed to address the test goals. Then, the second HTB queue is responsible to shape the data based on the current link data rate.

The control mechanism updates the dequeue rate $D Q_{r}$, resulting in increasing or decreasing inter-packet delay at the sender. The computation of $D Q_{r}$ uses the buffer occupancy $\Delta B$ and the data rate $\Delta d$ reported by the radio. Moreover, 
the user can define the buffer threshold $b$, the buffer warning area $w_{b}$, and the percentage of data rate reduction $r_{d}$. Eq. (8) shows the conditions to change the QDISC rules shaping the data flow whenever the network changes, therefore, controlling the buffer usage.

$$
D Q_{r}= \begin{cases}\max \left(\min (\Delta d), \Delta d * r_{d}\right) & \text { if } \Delta B \geq b \\ \max (\Delta d) & \text { elif }|\Delta B-b|>w_{b} \\ \Delta d & \text { otherwise }\end{cases}
$$

Let us explain how this mechanism works in three examples: 1) suppose the current data rate is $\Delta d=4.8 \mathrm{kbps}$, the max data rate supported is $\max (\Delta d=9.6 \mathrm{kbps})$, the $\min (\Delta d=100 \mathrm{bps})$, the buffer threshold is $b=50 \%$, the warning area is $w_{b}=10 \%$ and the current buffer occupancy is $\Delta B=20 \%$. Then, the second condition is satisfied $(|20-50|>10)$ and the HTB bitrate will set to the maximum data rate supported by the radio. The goal is to fill up the buffer as faster as the radio supports before the network condition gets worst; 2) now, the buffer occupancy reached $\Delta B=41 \%$, then the third condition is satisfied $(|41-50|<10)$ and the dequeue rate is set to $D Q_{r}=4.8 \mathrm{kbps}$, reducing the delivery rate from the queue to the radio buffer; 3 ) finally, if $\Delta B \geq 50 \%$, then the first condition will reduce the current $D Q_{r}$ in $w_{b}=10 \%$ until the buffer occupancy keeps lower than the threshold. In short, the control mechanism changes the dequeue rate like $D Q_{r}=\{4.8 \mathrm{kbps}, 4.32 \mathrm{kbps}, 3.88 \mathrm{kbps}, 3.49 \mathrm{kbps}, \ldots, 100 \mathrm{bps}\}$ until the buffer usage $\Delta B$ is close to the pre-defined threshold. If the link data rate change, the system will adapt its dequeue rate $D Q_{r}$ in a similar way. TNT was designed to test such a control mechanism within military systems over a wide range of communication scenarios.

\section{E. Monitoring and data analysis}

Having everything needed to execute an experiment, the next step is to deploy a monitoring and data analysis mechanism as shown in Fig. 1 (4). TNT process the data gathered from both sender and receiver nodes and the trace files, analyzing and visualizing these data together. The monitoring and data analysis phase was designed to allow to quantitatively understand the experiment, helping to highlight issues regarding the test environment and to improve systems such as routing protocols, military middlewares, as well as the QoS, required for a given application. Next, we describe each step of such a phase.

Data acquisition: The data acquisition process plays an important role to monitor the network state. This process was developed in our previous investigation [8], collecting all contextual data of both sender and receiver nodes such as IP packets, radio features (buffer and modulation) and store them in a centralized database which TNT uses to conduct further analysis.

Data processing: To analyze the military system performance, TNT process the IP packets from both sender and receiver nodes, sorting and combining them, getting the temporal series of packets received. With the time series, TNT process the data extracting the network metrics, such as data rate, delay,

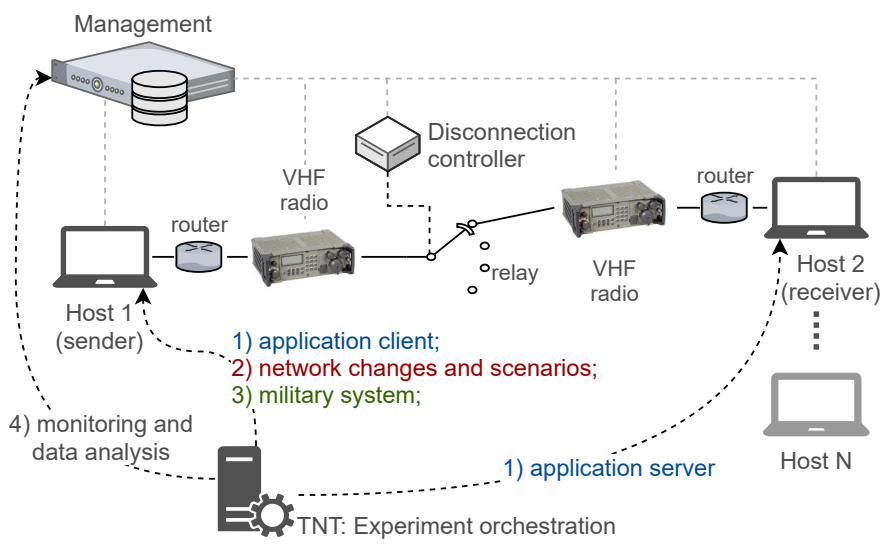

Fig. 4: Test environment.

jitter, and packet loss. Next, TNT exports the statistics in two different files: one compiling the overview of the experiment with basics statistics, such as min, max, average, and standard deviation, and the second file shows the entire log in a time series.

Visualization: Lastly, TNT plots the quantitative results after each experiment. The goal is to support the user in making decisions regarding the test environment, the user data flow (application layer), the network condition (physical layer), and the military systems (application, transport, and IP layers). In this sense, TNT gathers the files exported in the last stage combined with the traces files with mobility statistics and shows them graphically, as shown later in Section IV.

\section{EVALUATION}

In this section, we discuss quantitative results from different experimental configurations selected to demonstrate TNT supporting the tests of an exemplary military system over ever-changing scenarios. The experiments are conducted in a VHF network over seven network scenarios generated by different models. First, we describe the test environment and the user data flows. Then, we move to the experimental results, changing the network using model $\mathfrak{M}_{B 1}$ and model $\mathfrak{M}_{B 2}$, as discussed earlier in Section III.

\section{A. Test environment}

TNT was instantiated to perform experiments over real military radios, thus the infrastructure consists of VHF radios, Linux QDISC, the link disconnection prototype and a data monitoring and analysis process to measure the data sent and received, computing the packets and the trace statistics, as illustrated in Fig. 4. In this figure, TNT orchestrates the experiment by deploying all necessary scripts over the network nodes right before the experiment starts. After the user configures the experiment (as described earlier in Section III-A), TNT starts the experiment deploying (1) the application on the sender and receiver nodes (user data flows); (2) the network changes mechanism and the network scenarios based on mobility traces 


\begin{tabular}{ll}
\hline Components & Description \\
\hline Radio & 2 PR4Gs supporting $\{0.6,1.2,2,4,4,8,9.6\} \mathrm{kbps}$ \\
$\begin{array}{l}\text { Nodes } \\
\text { Management }\end{array}$ & 2 i7, 8 GB RAM laptops \\
$\begin{array}{l}\text { Link } \\
\text { disconnection }\end{array}$ & $\begin{array}{l}\text { Raspberry-Pi 3; Relay }(\mathrm{CX} 230 \mathrm{~L}) ; \\
\text { prototype }\end{array}$ \\
\hline $\begin{array}{l}\text { Step-up DC-DC Converter; 50-ohm attenuator } \\
\text { User data flow }\end{array}$ & $\begin{array}{l}\text { 2.000 UDP packets of 1264 Bytes MTU each, } \\
\text { following a uniform distributions } \eta_{u}=(a, b) \\
\text { where } a=1 \text { and } b=50 \text { packets per message/s }\end{array}$ \\
$\begin{array}{l}\text { Network } \\
\text { scenarios }\end{array}$ & $\begin{array}{l}7 \text { scenarios during } 170 \text { min each using model } \\
\text { Communication } \\
\text { area }\end{array}$ \\
\hline
\end{tabular}

TABLE I: Experimental setup

in the sender node, which has an interface with the radio and with the link disconnection prototype; (3) the military system (here the adaptive data flow shaping mechanism) on the sender node in order to avoid buffer overflow; and (4) the monitoring and data analysis which collect, prepare, processing and visualize the experiment outputs as describes later in this section.

The tactical network is composed of two VHF radios (PR4G), with $128 \mathrm{~kb}$ of buffer size and supporting five data rates $\{0.6,1.2,2,4,4,8,9.6\} \mathrm{kbps}$, each connected to a node (sender and receiver, respectively). The radio antennas are wired and connected to a link disconnection prototype in order to simulate the state $\{0\}$, disconnection. Then, the network condition is changed using mobility traces as described in Section III-C. Moreover, we have a server, in a management network, with methods to conduct the data monitoring and analysis, acquiring, processing, and plotting all logs. In the next sections, we describe the user data flow and the patterns of data rate changes used in the experiments, then discussing the experimental results. The experimental setup with all components used by TNT is described in Table I.

\section{B. User data flows}

The data flow can be generated in a variety of ways such as using specific applications in order to evaluate the QoS, or using a traffic generator designed to create different data flows. TNT uses the second approach to create traffic flows that can challenge military systems or simulate different applications based on different distributions of data flows. For example, some widely used traffic generators are Iperf, Netperf, Distributed Internet Traffic Generator (D-ITG), MultiGenerator (MGEN) [30]. Traffic generator should support stochastic models for packet size and inter departure time to simulate user data flows. These features can be found in the D-ITG tool as described in [31], [32] and for this reason TNT used it to generate and measure the user data flows in all experiments reported in this section. However, TNT is built in modules making easy the use of different traffic generators tools, specific application, or a new tool built using model $\mathfrak{M}_{A}$.

The user data flows used in the set of experiments discussed in this paper were generated with an instance of our model
$\mathfrak{M}_{A}(\eta, \theta, \omega, \mu)$. Meaning that TNT configured D-ITG to send a total of $\eta=2000$ UDP (User Datagram Protocol) packets of $\omega=1264$ Bytes (radio's MTU) each. Moreover, each message has a set of packets distributed following an uniform distribution and the delay between messages is $\theta=1 \mathrm{~s}$. The distribution of packets per messages is defined as $\eta_{u}=(a, b)$ with the interval $a=1$ and $b=50$ packets, where given the number of packet $x$ and $a \leq x \leq b$ the probability density function $P D F(x)=0.020$, meaning equal probability to have different number of packet (between 1 to 50) sent per message per second. Notice that, we defined $\eta$ and $\omega$ in order to ensure that the data will be sent during the whole mobility which takes about $170 \mathrm{~min}$. Besides, the goal is to demonstrate the TNT functionalities, testing the queuing discipline (part of a multi-layer military system) over a load of packets that surely demands a store-and-forward mechanism to successfully arrive at the receiver.

\section{Experiments over model $\mathfrak{M}_{B 1}$}

The model $\mathfrak{M}_{B 1}$ creates mobility patterns from a sequence of data rates supported by our VHF radios, as described earlier in Section III-C1. These data rates represent the boundaries of communication radius, meaning that changing the modulation we are implicitly moving the node in space and time. In order to create the ever-changing network conditions, we started with a Markov probability matrix (9) illustrated in Fig.5a. The matrix indexes matches the node states and it is configured to have low probabilities at states $\{0,4$, and 5$\}(0.15,0.1$, and 0.1$)$ and high probabilities at states $\{1,2$, and 3$\}(0.2$, 0.2 , and 0.25 ), where states $\{0,1,2,3,4,5\}$ correspond to $\{$ disconnection $\},\{0.6,1.2,2.4,4.8,9.6\} \mathrm{kbps}$ respectively. Notice that this particular configuration was arbitrarily chosen to highlight TNT's features. Varying these probabilities will change the pattern of changes as well as the mobility trace.

Thus, giving this matrix as an input to the model $\mathfrak{M}_{B 1}$ a sequence of methods are used to create three mobility patterns named Markov Raw (MR), Markov Filled (MF), and Markov Shortest Filled (MSF), as shown in Fig. 5. The principles to create these patterns were explained earlier in Section III-C1. To create the mobility patterns, model $\mathfrak{M}_{B 1}$ receives a sequence of states between $s_{0}$ and $s_{5}$ generated from the probability matrix (9), in this case study $s=30$. Moreover, the node movement starts at $s_{s t}=300 \mathrm{~s}$ and the time interval to state change was set to $s_{t i}=360 \mathrm{~s}$ seconds. These conditions directly impact the node mobility statistics as discussed later in this section. The MR, Fig. 5b, shows how the node is distributed over space with its respective network states, similarly, the patterns MF and MSF are shown in Fig. 5c and Fig. 5d, respectively. For the last two traces, TNT interpolates the positions with $n_{p}=10$, creating smooth

$$
\text { Markov }=\left(\begin{array}{ccccccc}
0 & 1 & 2 & 3 & 4 & 5 & \\
0.15 & 0.2 & 0.2 & 0.25 & 0.1 & 0.1 & 0 \\
0.15 & 0.2 & 0.2 & 0.25 & 0.1 & 0.1 & 1 \\
0.15 & 0.2 & 0.2 & 0.25 & 0.1 & 0.1 & 2 \\
0.15 & 0.2 & 0.2 & 0.25 & 0.1 & 0.1 & 3 \\
0.15 & 0.2 & 0.2 & 0.25 & 0.1 & 0.1 \\
0.15 & 0.2 & 0.2 & 0.25 & 0.1 & 0.1
\end{array}\right)
$$




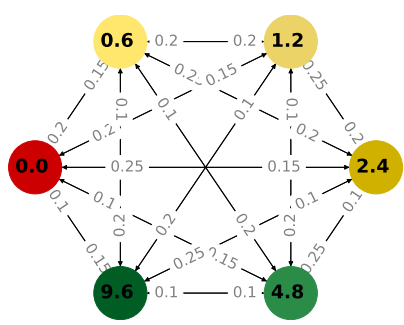

(a) Markov probabilities

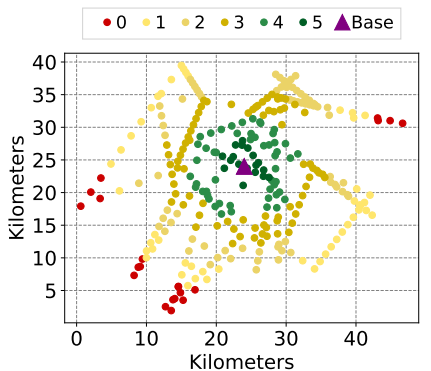

(c) Markov Filled

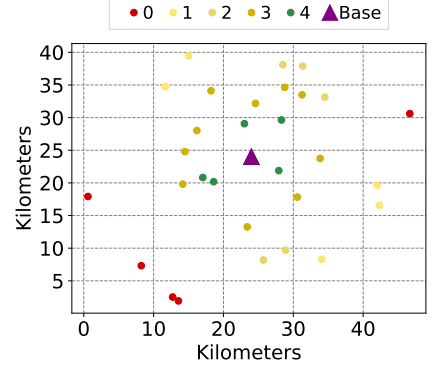

(b) Markov Raw

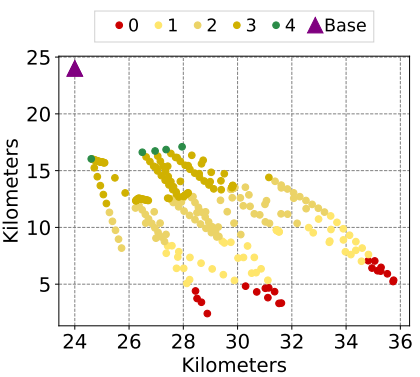

(d) Markov Shortest Filled
Fig. 5: From Markov chain to mobility pattern.

movements over time. In addition, for the trace MSF, TNT modify the distributions of states over the communication rings, choosing the closest location based on the last position, then creating short movements.

In order to better understand these mobility patterns Fig. 6 shows the pattern of changes in a time series and the overall statistics of each trace. Taking a look at the changes over time in Fig. 6a, we can see the difference among the patterns, even though, they were generated with the same probability matrix and sequence of states. This fact shows that mobility should be considered in the discussion of experimental results enriching the arguments to explain such test scenarios, otherwise the experiments can be interpreted or reproduced in different manners. In the MR trace, we can observe rapid state changes, jumping from one data rate to another. This pattern avoids any transitions between states, making it hard for systems to predict the network condition by monitoring the changes over time and acting to reduce packet loss. On the other hand, the MF trace shows smooth transitions between states interpolating them. Based on smooth movements, systems can sense small and slow network changes, that can be used as input to models designed to predict movements that increase the probability of packet loss. Finally, the MSF trace combines smooth movements and short distances. Notice that this trace reduces the node transitions because the node position is chosen not considering all possible places inside the ring area but the closest one from its last position.

The difference among these mobility patterns can be observed from the trace statistics as well. Fig. $6 \mathrm{~b}$ shows these

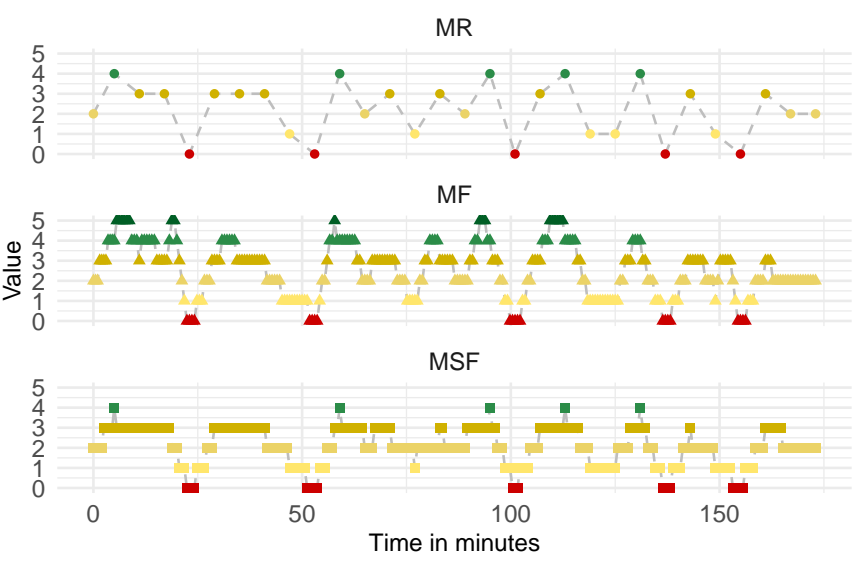

(a) Link data rates as a function of time.

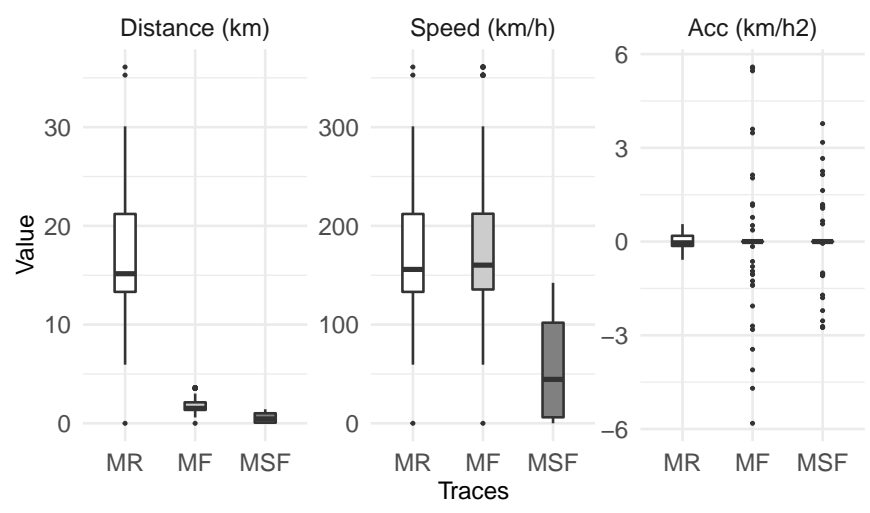

(b) Traces Statistics

Fig. 6: Traces created from the probability matrix in (9).

mobility statistics such as the distance between node positions, where the MR shows the largest traveled distances compared to the other patterns, reaching a maximum distance of $36 \mathrm{~km}$ and an average of $16.8 \mathrm{~km}$ traveled between positions. The speed metric of MR and MF traces show almost the same variation, reaching a max speed up to $360 \mathrm{~km} / \mathrm{h}$ and averaged speeds about $169 \mathrm{~km} / \mathrm{h}$ and $174 \mathrm{~km} / \mathrm{h}$, respectively. This is due to the interpolation process that divides the distance and time filling the transition states proportionally, resulting in the same node speed. Differently, the trace MSF is a result of the shortest distance approach, showing lower speed in average $58 \mathrm{~km} / \mathrm{h}$, compared to the other patterns. The difference can also be noticed in the acceleration metric, where we can see in MR and MF traces a high average acceleration of $0.01 \mathrm{~km} / \mathrm{h}^{2}$ compared to MSF with $0.001 \mathrm{~km} / \mathrm{h}^{2}$. Table II shows the overview of the three patterns of changes presented using Markov chain. In addition to the mobility metrics, this table also shows the amount of variation, in terms of the nominal data rate, each trace can supports. In other words, MF supports the highest data rate of $2.5 \mathrm{kbps}$ averaged, and MSF has the lowest rate 


\begin{tabular}{lccccccc}
\hline \multirow{2}{*}{ Traces } & \multicolumn{8}{c}{ Features } \\
\cline { 2 - 8 } & $\begin{array}{c}\text { Data rate }- \text { Kbps } \\
\text { (avg } \pm \mathrm{sd})\end{array}$ & \multicolumn{2}{c}{ Distance $-k m$} & \multicolumn{2}{c}{ Speed $-k m / h$} & \multicolumn{2}{c}{ Acc. $-k m / h^{2}$} \\
\cline { 2 - 8 } & $1.9 \pm 1.59$ & Max & Avg & Max & Avg & Max & Avg \\
\hline MR & $2.5 \pm 2.35$ & 3.6 & 16.8 & 360 & 169 & 0.55 & 0.01 \\
MF & $1.5 \pm 0.91$ & 1.4 & 0.57 & 142 & 58 & 3.7 & 0.001 \\
MSF & $1.5 \pm 0.5$ &
\end{tabular}

TABLE II: Trace statistics created from a Markov model.

of $1.5 \mathrm{kbps}$ averaged. Later, we show the impact of such a condition on experimental results.

As mentioned before, the test can be done over different conditions even though it follows the same sequence of states. Therefore, a test scenario characterization through the mobility metrics plays an important role to comprehend such a scenario, its goals, and limitations. For example, these patterns can simulate different entities in a field such as vehicles and humans patrolling a given area. The MSF trace could be characterized as a vehicular entity covering small areas with an average speed of $58 \mathrm{~km} / \mathrm{h}$ in an uneven area, requiring high variation on acceleration. The MR could characterize a nonhuman movement as an unmanned aerial vehicle, covering larger distances and reaching high speeds. Notice that, the idea to create models to change the network topology reflecting nodes mobility is to allow the characterization of such test scenarios. Moreover, using models we can create different test scenarios by modifying a set of parameters that can be reproduced for independent verification.

1) Test-bed experiment: Before starting the experiment, TNT deploys the application, that creates data flows, and the network scenarios in our real environment. Fig. 7 shows the experimental results through different network metrics such as the radio data rate (Radio $D R$ ), the data rate computed at the receiver side $(D R)$, the radio buffer (Buffer), the packet loss (P. Loss), the end-to-end delay (Delay), and jitter (Jitter). Notice the Radio DR and Buffer metrics were acquired from the VHF radio through SNMP and compiled together with the other metrics to show the overall view of the experiment. The radio data rate is the nominal data rate in use and represents the changes TNT creates using the mobility traces, this feature is also shown in Fig. 6a.

Based on this experiment, we are able to test the adaptive shaping mechanism (military system), described in Section III-D, through a user data flow over different network conditions. The Radio DR metric shows how the network changes during about $170 \mathrm{~min}$, and then the node stop "moving". However, the data flow still going through the radio link until the queue and the buffer is empty. This behavior is explained by the throughput supported by the network scenarios, but also by the shaping mechanism adding inter-packet delays to avoid buffer overflow. Therefore, each network scenario takes different time intervals to end, for instance, MF takes about $346 \mathrm{~min}$, MR about $365 \mathrm{~min}$, and MSF takes about $417 \mathrm{~min}$. Notice that, the $D R$ is kept below the Radio DR because the radio specifies a nominal modulation, as mentioned before, but the real link data rate can only be computed at the receiver node, and the values are always lesser than the nominal data rates. Thus, MF can support a data rate of $1 \mathrm{kbps}$ on average,

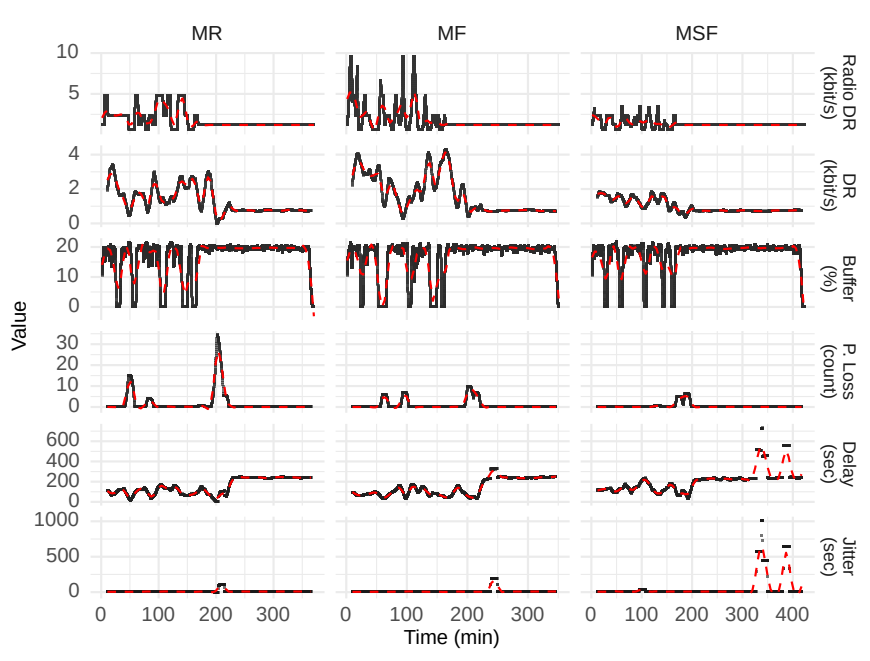

Fig. 7: Network statistics over Markov mobility traces.

\begin{tabular}{|c|c|c|c|c|}
\hline \multirow{2}{*}{\multicolumn{2}{|c|}{ Metrics }} & \multicolumn{3}{|c|}{ Traces } \\
\hline & & MR & MF & MSF \\
\hline \multicolumn{2}{|c|}{$\begin{array}{l}\text { Experiment } \\
\text { duration (min) }\end{array}$} & 365.25 & 346.19 & 417.25 \\
\hline \multicolumn{2}{|c|}{$\begin{array}{l}\text { Data Rate in kbps } \\
\text { (avg } \pm \text { sd) }\end{array}$} & $0.98 \pm 0.72$ & $1 \pm 0.95$ & $0.8 \pm 0.7$ \\
\hline \multirow{4}{*}{ Packets } & Sent & 2000 & 2000 & 2000 \\
\hline & Recv. & 1796 & 1851 & 1869 \\
\hline & Drop. & $10.2 \%$ & $7.4 \%$ & $6.5 \%$ \\
\hline & Rate (avg/s) & 0.08 & 0.09 & 0.07 \\
\hline \multirow{3}{*}{$\begin{array}{l}\text { Delay } \\
(\mathrm{sec})\end{array}$} & Min & 5.8 & 6.2 & 5.1 \\
\hline & Max & 2899.7 & 5878.13 & 19519.66 \\
\hline & $\operatorname{Avg} \pm \mathrm{sd}$ & $171 \pm 101$ & $157 \pm 157$ & $221 \pm 673$ \\
\hline Jitter ( $\mathrm{sec}$ & $\operatorname{Avg} \pm \mathrm{sd}$ & $11.2 \pm 96.3$ & $13.4 \pm 185$ & $62.7 \pm 943$ \\
\hline
\end{tabular}

TABLE III: Statistics from experiments over Markov traces.

while MR supports $0.98 \mathrm{kbps}$, and MSF $0.8 \mathrm{kbps}$ on average.

The results of the adaptive shaping mechanism are better visualized in the Buffer metric, where we can see the data flow keeping very close to the defined threshold of $b=20 \%$, avoiding buffer overflow and reducing as much as possible packet loss. Moreover, the metrics Delay and Jitter also reflect the behavior of the mechanism, showing delays added between packets and how it varies over time. The observed minimum delay in our VHF network, for all scenarios, is higher than $5.1 \mathrm{~s}$. The average delays observed in MF was $157 \mathrm{~s}$, MR about $171 \mathrm{~s}$, and $221 \mathrm{~s}$ in MSF scenario. Moreover, the jitter metric shows the highest variation per second about $62 \mathrm{~s}$ in MSF, while MR has the lowest value about $11 \mathrm{~s}$. These metrics describe the boundaries of QoS for a given communication scenario, helping to identify which type of application can be used in such a scenario.

These experiments show the capabilities of our radios and how much variation in the link data rate (mobility) it can support. The changes on the MR happen every $s_{t i}=360 \mathrm{~s}$ jumping from one state to another, where we can see more 
packet loss reaching about $10 \%$. Due to the smooth state transitions (smooth movements), we noticed $7.4 \%$ and $6.5 \%$ of packet loss over smooth MF and over shortest movements MSF. This is explained by the mechanism implemented in our adaptive shaping, aiming to detect abnormal buffer behavior such as flushing all data stored during a disconnection time. Thus, these experiments show that our VHF radios cannot support too many and long disconnections over time, about $360 \mathrm{~s}$, thus requiring system support like the adaptive shaping mechanism to minimize packet loss.

We also noticed that these changes are strongly related to the routing protocol used in the radios to perform neighbor discovery and its configuration parameters, in our case OLSR, which cannot be manipulated without a radio manager system with proper permission. Once the flush happens, resulting in packet loss, the radio also takes time to resume IP data flows, adding more delays and degrading QoS. Therefore, the military system needs to act to minimize the impact of link disconnections and the resulting flush in the radio buffer. The shaping mechanism minimizes this issue by defining two rules (i) a low buffer threshold of $b=20 \%$ (could be less), in order to reduce the packet loss in case of flushing; (ii) identifying these flushes on the buffer and prevent more loss reducing the dequeue delivery rate to a minimum as possible. Table III summarizes the experimental results discussed in this section. Thus, after the experiment ends TNT exports a summary as shown in this table in addition to the result visualized in a time series.

\section{Experiments over model $\mathfrak{M}_{B 2}$}

Now we describe the experiments using model $\mathfrak{M}_{B 2}$ which reuses well-known mobility models to create patterns of network change as described earlier in Section III-C2. We set TNT to use BonnMotion to generate four exemplary mobility patterns, from four different models, namely Gauss-Markov (GM), Random Waypoint (RWP), Manhattan Grid (MG), and Probabilistic Random Walk (PRW). Fig. 8, shows how the node is distributed over space and its respective network states. Notice that model $\mathfrak{M}_{B 2}$ is independent of mobility model generator BonnMotion and it supports any trace file with a format node $_{i}=[x, y$, time $]$.

To understand the mobility models and the trace characteristics generated by them, next, we briefly describe each model and the parameters configured to create a given mobility pattern. Thus, the GM model [33] is designed to adapts the node movement based on its current location, speed and direction. At a given time intervals these parameters are updated creating the movement as function of time. Fig. 8a shows the mobility pattern simulating node movement through an uneven terrain. This trace was generated using the following parameters: frequency to update the movement $f_{u}=20 \mathrm{~s}$, angle deviation $a_{s t d}=0.39$, speed deviation speed $_{\text {std }}=0.5 \mathrm{~m} / \mathrm{s}$, and maximum speed $\max _{s}=16.7 \mathrm{~m} / \mathrm{s}(60 \mathrm{~km} / \mathrm{h})$. MG model [34] uses a grid road topology looking like urban area as shown in Fig. 8b. It is mostly used for tests in vehicular networks where mobile nodes move on streets of a city, following a given probability to turn or keep straight on in each intersection.

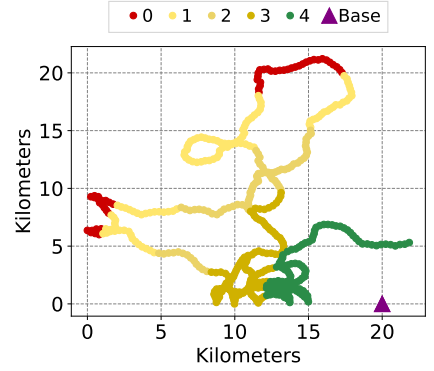

(a) Gauss Markov.

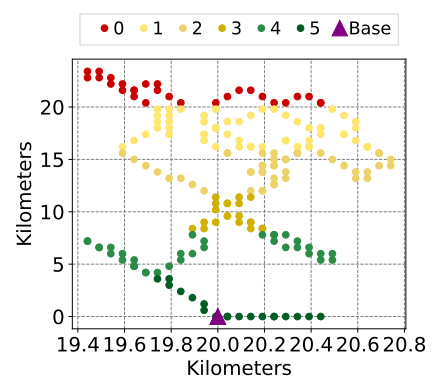

(c) Probabilistic Random Walk.

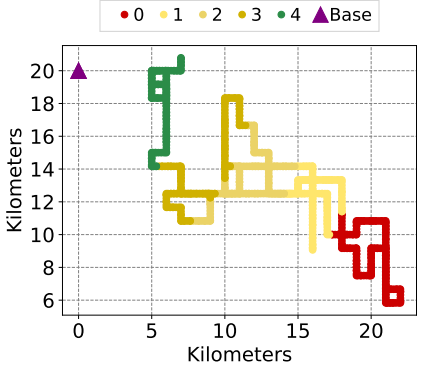

(b) Manhattan Grid.

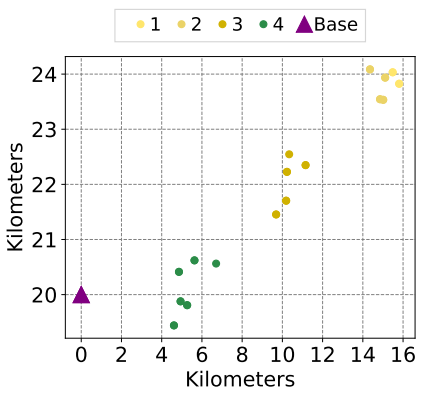

(d) Random Waypoint
Fig. 8: Mobility pattern from mobility models.

We configured the model with the following probabilities: the mobile node goes straight with a probability of $f=0.5$ and turn left with $t_{l}=0.25$ or turn right with $t_{r}=0.25$. We also defined the minimum speed of $\min _{s}=0.1 \mathrm{~m} / \mathrm{s}(0.36 \mathrm{~km} / \mathrm{h})$, the maximum averaged speed about $\max _{a s}=16.7 \mathrm{~m} / \mathrm{s}$, a pause probability of $p_{p}=0.1$ (if there is no speed changes) and a pause time about $p_{t}=30 \mathrm{~s}$.

PRW [28] chooses new directions and speeds to travel based on a pre-defined probabilities. This model, shown in Fig. 8c, uses a Markov probability matrix to define the probabilities to move in any direction at a fixed speed or remaining still on that direction. Moreover, this model creates erratic movement with a given probability, simulating the unpredictable movement of many entities in nature. Lastly, we used the RWP model [35] and the generated trace is shown in Fig. 8d. The node starts without moves for a certain period of time $p_{t}=600 \mathrm{~s}$, then it chooses a random destination. Its speed follows an uniform distribution and we defined the minimum speed about $\min _{s}=10 \mathrm{~m} / \mathrm{s}(36 \mathrm{~km} / \mathrm{h})$ and maximum speed of $\max _{s}=30 \mathrm{~m} / \mathrm{s}(108 \mathrm{~km} / \mathrm{h})$. Once the node reaches the pre-defined checkpoints such as $c p_{1}(5 \mathrm{~km}, 20 \mathrm{~km})$, $c p_{2}(10 \mathrm{~km}, 22 \mathrm{~km})$, and $c p_{3}(15 \mathrm{~km}, 24 \mathrm{~km})$ it pauses for $p_{t}$ seconds before starting the process again.

Complementing Fig. 8, Fig. 9a, shows the distribution of link data rates over time for the four mobility models. These patterns can represent different entities moving in a battlefield/scenario as described before, enriching the discussion and explanation of given system behavior based on a network scenario (node mobility). The difference among these patterns 


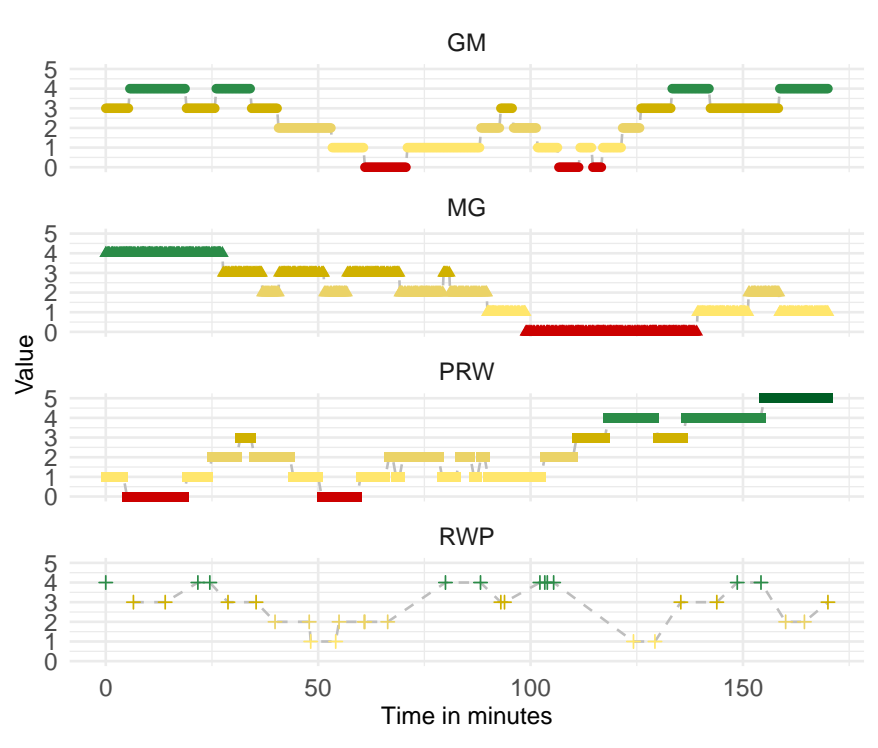

(a) Link data rates as a function of time.

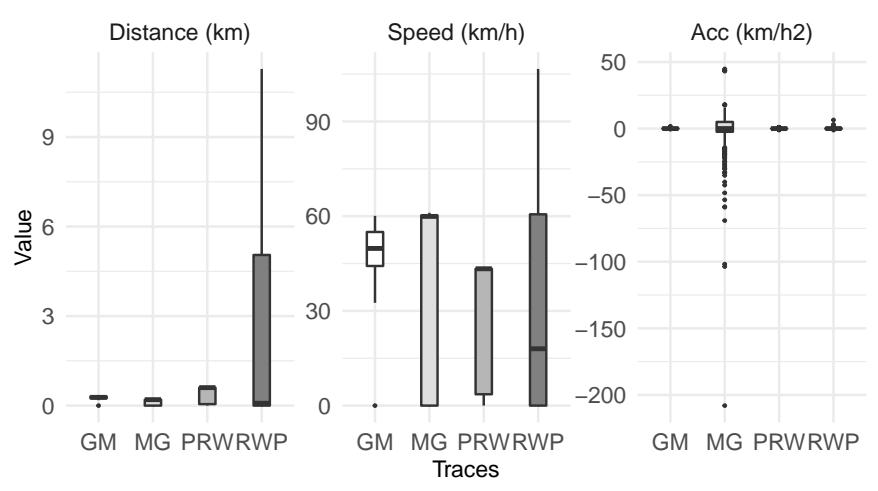

(b) Traces Statistics.

Fig. 9: Traces created from mobility models.

is also observed on the trace statistics shown in Fig. 9b. Comparing the distance, speed, and acceleration features, it is noticed that the GM trace shows the most stable movements compared to the other patterns, with small variation around the mobility metrics with an average speed of about $47 \mathrm{~km} / \mathrm{h}$, acceleration about $0.005 \mathrm{~km} / \mathrm{h}^{2}$ averaged and distance traveled between positions about $0.27 \mathrm{~km}$ in average.

The node at MG can reach $40 \mathrm{~km} / \mathrm{h}$ and traveled distance about $0.12 \mathrm{~km}$ averaged with high averaged deceleration about $0.9 \mathrm{~km} / \mathrm{h}^{2}$, simulating a vehicle behavior in an urban area. The PRW trace shows long traveled distances about $0.44 \mathrm{~km}$, compared with the last traces, at low speed about $31 \mathrm{~km} / \mathrm{h}$ with no much variation in acceleration about $0.0003 \mathrm{~km} / \mathrm{h}^{2}$, all in average. For example, these attributes can be used to describe an unmanned patrol in a plateau terrain. Finally, the RWP trace introduces more variability in the traveled distances about $2.59 \mathrm{~km}$ averaged and reaching maximum speed of $108 \mathrm{~km} / \mathrm{h}$.

\begin{tabular}{lccccccc}
\hline \multirow{2}{*}{ Traces } & \multicolumn{9}{c}{ Features } \\
\cline { 2 - 8 } & $\begin{array}{c}\text { Data rate }- \text { Kbps } \\
\text { (avg } \pm \mathrm{sd})\end{array}$ & \multicolumn{2}{c}{ Distance $-k m$} & \multicolumn{2}{c}{ Speed $-k m / h$} & \multicolumn{2}{c}{ Acc. $-k m / h^{2}$} \\
\cline { 2 - 8 } & $2.1 \pm 1.7$ & Max & Avg & Max & Avg & Max & Avg \\
\hline GM & $1.6 \pm 1.6$ & 0.2 & 0.12 & 61 & 40 & 44 & 0.005 \\
MG & $2.4 \pm 2.7$ & 0.6 & 0.44 & 43 & 31 & 0.8 & 0.0003 \\
PRW & 2.4 & 11.2 & 2.59 & 106 & 30 & 6.4 & 0.31 \\
RWP & $2.7 \pm 1.6$ & &
\end{tabular}

TABLE IV: Trace statistics created from mobility models.

Moreover, this trace has specific checkpoints (areas) where it stay for while before starts move again, which can be characterized as vehicular movements, based on the speed and distances the entity can reach. Table IV shows the numeric overview regarding the mobility traces MG, PRW, GM and RWP discussed in this section.

1) Test-bed experiment: Following the same methodology described in the experimental results with model $\mathfrak{M}_{B 1}$. Here, the adaptive shaping mechanism is tested over the network conditions provided by the model $\mathfrak{M}_{B 2}$ as illustrated in Fig. 10 by different network metrics. The first observation is the experiment duration, where MG took about $536 \mathrm{~min}$ (more then 8 hours) to end the experiment, the GM and glsrwp took about $210 \mathrm{~min}$, and PRW $197 \mathrm{~min}$. This is explained by the average data rate and the duration of disconnections in each trace. It is also visible that our shaping mechanism can hold the user data flow in order to keep the radio buffer occupancy (Buffer) close to the pre-defined threshold of $b=20 \%$. Moreover, in all patterns, except RWP, an abnormal radio behavior with buffer flushing is noticed, converging to the same explanation done in Section IV-C regarding the limitations of our VHF radios. Even though this behavior could negatively impact the packet delivery, our shaping mechanism was able to sense these abrupt changes on the buffer occupancy and adapt the dequeue rate to the minimum as possible, reducing packet loss while the route is not recovered. Therefore, the minimum packet loss $(P$. Loss) observed in MG reached about $24 \%$, PRW with $4.8 \%$ and GM reaching about $3.7 \%$ of packet loss. Although RWP does not contain disconnections, we observed some packets loss of about $1.6 \%$ which can be explained by the probability of packet loss increases in low data rates as calculated in [3] using experimental results with the same VHF radios.

Regarding the end-to-end delay (Delay) the minimum delay observed in our VHF network was $4.17 \mathrm{~s}$. In average, PRW shown the minimum value about $95 \mathrm{~s}$ compared to GM with $101 \mathrm{~s}$, RWP $112 \mathrm{~s}$, and MG reaching 199s in average. The Jitter metric of PRW reached the highest variation, about $21 \mathrm{~s}$, followed by MG with $12 \mathrm{~s}$, GM with $7.4 \mathrm{~s}$ and RWP reaching about $5.7 \mathrm{~s}$, all in average. Summarizing the discussion above, Table V shows the quantitative results of our proposed military system over the mobility patterns of model $\mathfrak{M}_{B 2}$.

\section{Discussion}

The development of robust and reliable military systems depends on the development of multi-layer control mechanisms tested over a wide range of communication scenarios. However, currently, the process of test military systems lacks the element of chance restricting the evaluation to a simple 


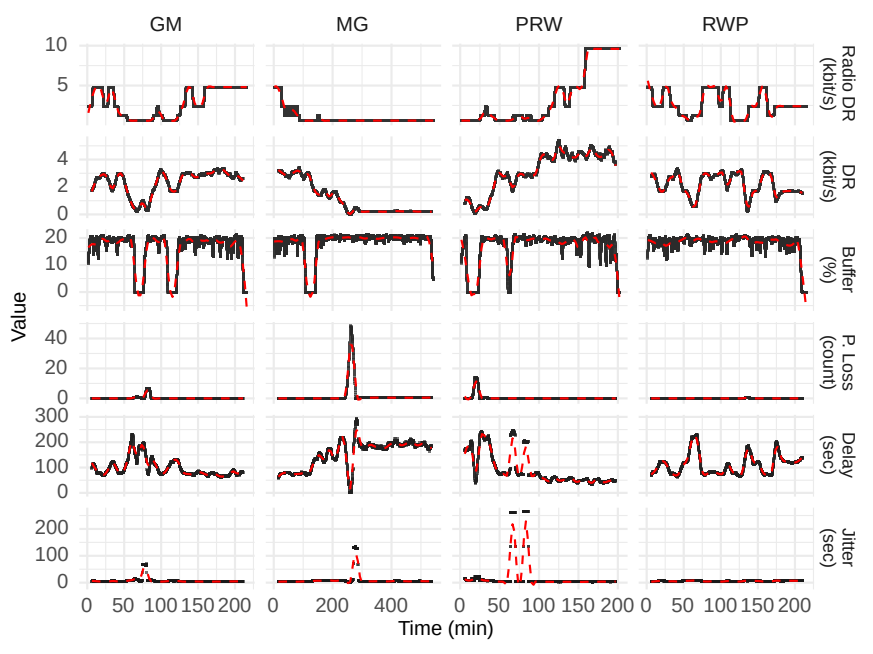

Fig. 10: Network statistics over mobility traces.

\begin{tabular}{|c|c|c|c|c|c|}
\hline \multirow{2}{*}{\multicolumn{2}{|c|}{ Metrics }} & \multicolumn{4}{|c|}{ Traces } \\
\hline & & GM & MG & PRW & RWP \\
\hline \multicolumn{2}{|c|}{$\begin{array}{l}\text { Experiment } \\
\text { duration }(\mathrm{min})\end{array}$} & 210.86 & 536.59 & 197.26 & 210.061 \\
\hline \multicolumn{2}{|c|}{$\begin{array}{l}\text { Data Rate in kbps } \\
\text { (avg } \pm \text { sd) }\end{array}$} & $0.97 \pm 0.75$ & $0.79 \pm 1.1$ & $0.87 \pm 0.96$ & $2.1 \pm 1.5$ \\
\hline \multirow{4}{*}{ Packets } & Sent & 2000 & 2000 & 2000 & 2000 \\
\hline & Recv. & 1925 & 1513 & 1904 & 1968 \\
\hline & Drop. \% & $3.75 \%$ & $24.35 \%$ & $4.8 \%$ & $1.6 \%$ \\
\hline & Rate (avg/s) & 0.15 & 0.04 & 0.16 & 0.15 \\
\hline \multirow{3}{*}{$\begin{array}{l}\text { Delay } \\
(\mathrm{sec})\end{array}$} & Min & 10.04 & 5.29 & 4.17 & 14.24 \\
\hline & Max & 2023.4 & 3889.2 & 7900.5 & 344.02 \\
\hline & Avg \pm sd & $101 \pm 67$ & $199 \pm 144$ & $95 \pm 260$ & $112 \pm 54$ \\
\hline Jitter $(\mathrm{sec})$ & Avg \pm sd & $7.4 \pm 59$ & $12 \pm 131$ & $21 \pm 355$ & $5.7 \pm 5.9$ \\
\hline
\end{tabular}

TABLE V: Statistics from experiments over mobility traces.

set of scenarios with static or periodic changes. To the best of our knowledge this could be explained by (i) the inherent security issues in this field, restricting the general scientific community to access classified investigations that may have better test methodologies; (ii) the complexity and high-costs to create different test scenarios in reality; the lack of models to emulate military communication technologies in virtualized environments; investigations lacking scenario characterization to ensure that the experiments are stressing the military systems being tested. Therefore, this paper complements the stateof-the-art by introducing a test platform for tactical networks (TNT) to quantify the robustness of military systems over everchanging scenarios.

TNT connects three main components (i) user data flows, (ii) network scenarios, and (iii) military system, building and wrapping all necessary methods (models for eve-changing scenarios, an exemplary military system, data parsers, monitoring and data analysis, and a link disconnection prototype) to provide an easy-to-use test platform. The first component aims to address the issue of limited data flows experimented over the test scenarios. Usually, the investigations in the literature use specific data flows from a particular application with little or no randomness on the diversity of QoS requirements. Handling this issue, TNT proposes a general model $\left(\mathfrak{M}_{A}\right)$ in order to demonstrate how wide the user data flow can be generated, simulating different applications by the manipulation of some parameters such as the number of packets, frequency, and size. The second component address the lack of chance in the network scenarios. Meaning that most experiments in the literature restrict the network scenario to static or periodic changes in the link quality (e.g. with link disconnections of 60 seconds). Some of them, introduce a certain level of network changes, but they are still missing a proper evaluation or discussion regarding the characterization of these changes. In this sense, TNT proposes different methods to create network scenarios based on model $\left(\mathfrak{M}_{B 1}\right)$ : a sequence of network states (link data rates), generated by stochastic models, where the user inputs the respective probabilities of network states; or model $\left(\mathfrak{M}_{B 2}\right)$ where the user defines which mobility model represents better the test scenario.

Moreover, we proposed a mobility model to translate any sequence of network states into mobility traces. This model opened the discussion that sequences of data rates to create network changes, as we previously investigated in [4], [5], [7]-[9], can follow different mobility patterns even though it comes from the same sequence of changes. Reproducing these changes in a real environment requires, in most cases, the addition of mobile nodes. Therefore, these nodes can have different mobility patterns depending on which entity is involved in the test, such as humans, ground vehicles, aerial vehicles, or unmanned vehicles. The node characterization will vary in its motion (speed, acceleration, and distance traveled) requiring different communication technologies (UHF, VHF and SatCom) and protocols (transport and routing protocols) to keep the connectivity.

Regarding the third component, current literature shows military systems adapting the user data flow to the network constraints in order to avoid buffer overflow and packet loss, due to the military radios are resource-constrained devices. However, most of the published solutions are not reproducible due to the lack of methodology describing the test scenarios in precise terms (say using reproducible models). Some solutions lack the shaping of multi-cast data flows, and others require an additional stack of software to operate properly. Therefore, the experimental results discussed in this article suggest that a control mechanism using Linux queue discipline and radio feedback can be scalable, reliable, and robust to changes in the link data rate. And TNT provides the tools to compile quantitative evidence supporting these claims.

Even though, this study addressed some challenges in testing tactical systems as mentioned before, there are still some open issues defined as follows: (i) currently, TNT simulate limited network scenarios with uncorrelated nodes movements, instead, in reality, military unities move in convoys, requiring the use of group mobility models to represent them. (ii) model $\left(\mathfrak{M}_{B 1}\right)$ does not support mobility metrics as input such as speed, distance. (iii) TNT still requires some expertise from users to configure the shaping mechanism following the radio constraints, such as the supported modulations, buffer size, and buffer threshold. In addition, the military radio must have an 
interface such as SNMP to enable the feedback loop.

\section{CONCLUSION}

This paper introduced a software platform (TNT) to test military systems over ever-changing communication scenarios. TNT generates scenarios with different patterns of change for network conditions and user data-flows, also collecting and analyzing data from experiments, and showing quantitative performance metrics. TNT wrap the necessary models to automate the evaluation of military systems and applications over real military radios. Therefore, TNT supports (i) the creation of a variety of data flows by the definition of a general model $\left(\mathfrak{M}_{A}\right)$ instantiated with a traffic generator tool; (ii) models $\left(\mathfrak{M}_{B 1}\right.$ and $\left.\mathfrak{M}_{B 2}\right)$ for changing network conditions through a stochastic mobility model, transforming the sequence of network states (link data rates) in a mobility pattern and mapping mobility traces to link data rates, allowing the use of mobility traces in stationary radios in a laboratory. TNT also include a prototype to create link disconnections in radios with wired antennas.

Addressing the problem of frequent changes in the link quality that may lead to buffer overflow in military radios, TNT introduced (iii) an adaptive mechanism to shape the user data flow to the network conditions; Finally, (iv) TNT's monitoring and data analysis scripts supported the discussion of experimental results from seven different scenarios, including plots and statistics, characterizing both the system's performance through network metrics and the changes in the communication scenario through mobility metrics.

As future work, we intend to extend TNT to support emulated network environments with SDN-capable devices and military communication technologies, such as VHF, UHF, and SatCom. The goal is to increase the scale of the test environment and improve military systems before its deployment in the real environment. Moreover, we plan to improve the network-changing model $\mathfrak{M}_{B 1}$ by introducing interference that simulates barriers along the mobility traces and extend it to support mobility metrics as input such as speed, distance, and non-linear movements.

\section{ACKNOWLEDGMENT}

The authors would like to thank Bundeswehr, the unified armed forces of Germany (BAAINBw and WTD81), for the several national and international grants supporting the development of the ideas converging to the present article.

\section{REFERENCES}

[1] G. Elmasry, "A comparative review of commercial vs. tactical wireless networks," IEEE Communications Magazine, vol. 48, no. 10, pp. 54-59, October 2010.

[2] R. Amin, D. Ripplinger, D. Mehta, and B. N. Cheng, "Design considerations in applying disruption tolerant networking to tactical edge networks," IEEE Communications Magazine, vol. 53, no. 10, pp. 32-38, October 2015.

[3] J. Loevenich, R. R. F. Lopes, P. H. Rettore, S. M. Eswarappa, and P. Sevenich, "Maximizing the probability of message delivery over ever-changing communication scenarios in tactical networks," IEEE Networking Letter, pp. 1-5, February 2021, (under review).
[4] R. R. F. Lopes, P. H. Balaraju, P. H. Rettore, and P. Sevenich, "Queuing over ever-changing communication scenarios in tactical networks," IEEE Transactions on Mobile Computing, pp. 1-15, June 2020.

[5] P. H. Balaraju, P. H. Rettore, R. R. F. Lopes, S. M. Eswarappa, and J. Loevenich, "Dynamic adaptation of the user data flow to the changing data rates in VHF networks: An exploratory study," in 11th IEEE International Conference on Network of the Future (NoF), Bordeaux, France, Oct 2020, pp. 64-72.

[6] R. R. F. Lopes, J. Loevenich, P. H. L. Rettore, S. M. Eswarappa, and P. Sevenich, "Quantizing radio link data rates to create ever-changing network conditions in tactical networks," IEEE Access, vol. 8, pp. 188 015-188 035, 2020.

[7] R. R. F. Lopes, P. H. Balaraju, and P. Sevenich, "Creating and handling ever-changing communication scenarios in tactical networks," in 15th International Conference on the Design of Reliable Communication Networks (DRCN), Coimbra, Portugal, March 2019, pp. 67-74.

[8] R. R. F. Lopes, P. H. Balaraju, A. T. Silva, P. H. Rettore, and P. Sevenich, "Experiments with a queuing mechanism over ever-changing datarates in a VHF network," in IEEE Military Communications Conference (MILCOM), Norfolk VA, USA, November 2019, pp. 131-136.

[9] R. R. F. Lopes, P. H. Balaraju, and P.Sevenich, "Creating ever-changing QoS-constrained dataflows in Tactical Networks: An exploratory study," in International Conference on Military Communications and Information Systems (ICMCIS), Budva, Montenegro, May 2019.

[10] N. Suri, A. Hansson, J. Nilsson, P. Lubkowski, K. Marcus, M. Hauge, K. Lee, B. Buchin, L. Mısırhoğlu, and M. Peuhkuri, "A realistic military scenario and emulation environment for experimenting with tactical communications and heterogeneous networks," in International Conference on Military Communications and Information Systems (ICMCIS), 2016, pp. 1-8.

[11] L. Prior, C. Fossa, D. Ward, Jun Sun, P. Boehm, E. Kuczynski, J. Cain, and T. Mak, "Tactical network integration test framework," in IEEE Military Communications Conference (MILCOM), 2011, pp. 21582163.

[12] K. M. Marcus, K. S. Chan, R. L. Hardy, and P. L. Yu, "An environment for tactical sdn experimentation," in IEEE Military Communications Conference (MILCOM), Los Angeles, CA, USA, Oct. 2018, pp. 1-9.

[13] Q. Zhao, A. J. Brown, J. H. Kim, and M. Gerla, "An integrated softwaredefined battlefield network testbed for tactical scenario emulation," in IEEE Military Communications Conference (MILCOM), Norfolk, VA, USA, Nov. 2019, pp. 373-378.

[14] iTrinegy, "Software defined test networks." [Online]. Available: https://itrinegy.com/solution-by-industry/military/

[15] A. Poylisher, C. Serban, J. Lee, T. Lu, R. Chadha, C. J. Chiang, K. Jakubowski, and R. Orlando, "Virtual ad hoc network testbeds for high fidelity testing of tactical network applications," in MILCOM 2009 - 2009 IEEE Military Communications Conference, 2009, pp. 1-7.

[16] S. Doshi, U. Lee, B. Bressler, R. Bagrodia, M. DiGennaro, J. Oleksa, and Y. Chen, "Operationally realistic testing of network centric tactical applications in a lab environment," in MILCOM 2012 - 2012 IEEE Military Communications Conference, 2012, pp. 1-6.

[17] N. Jansen, D. Krämer, and M. Spielmann, "Testbeds for it systems in tactical environments," in IEEE Military Communications Conference (MILCOM), 2014, pp. 1293-1298.

[18] C. Serban, A. Poylisher, A. Sapello, Y. Gottlieb, C. J. Chiang, and R. Chadha, "Testing android devices for tactical networks: A hybrid emulation testbed approach," in IEEE Military Communications Conference (MILCOM), 2015, pp. 489-494.

[19] A. Ghosh, S.-w. Li, C. J. Chiang, R. Chadha, K. Moeltner, S. Ali, Y. Kumar, and R. Bauer, "Qos-aware adaptive middleware (qam) for tactical manet applications," in IEEE Military Communications Conference (MILCOM), 2010, pp. 178-183.

[20] A. Poylisher, F. Sultan, A. Ghosh, S.-w. Li, C. J. Chiang, R. Chadha, K. Moeltner, and K. Jakubowski, "Qam: A comprehensive qos-aware middleware suite for tactical communications," in IEEE Military Communications Conference (MILCOM), 2011, pp. 1586-1591. 
[21] A. S. Peng, D. M. Moen, T. He, and D. J. Lilja, "Automatic dynamic resource management architecture in tactical network environments," in IEEE Military Communications Conference (MILCOM), 2009, pp. 1-7.

[22] T. Chen, S. Eswaran, M. A. Kaplan, S. Samtani, D. Shur, J. Sucec, and L. Wong, "Enhancing application performance with network awareness in tactical networks," in IEEE Military Communications Conference (MILCOM), 2011, pp. 1158-1163.

[23] R. Fronteddu, A. Morelli, M. Tortonesi, N. Suri, C. Stefanelli, R. Lenzi, and E. Casini, "Ddam: Dynamic network condition detection and communication adaptation in tactical edge networks," in IEEE Military Communications Conference (MILCOM), 2016, pp. 970-975.

[24] R. Fronteddu, A. Morelli, E. Casini, N. Suri, B. Jalaian, and L. Sadler, "A content and context-aware solution for network state exchange in tactical networks," in IEEE Military Communications Conference (MILCOM), 2017, pp. 430-435.

[25] A. Bujari, C. T. Calafate, J.-C. Cano, P. Manzoni, C. E. Palazzi, and D. Ronzani, "Flying ad-hoc network application scenarios and mobility models," International Journal of Distributed Sensor Networks, vol. 13, no. 10, p. 1550147717738192, 2017.

[26] S. Kumar, S. Sharma, and B. Suman, "Mobility metrics based classification \& analysis of mobility model for tactical network," International Journal of Next-Generation Networks (IJNGN), vol. 2, no. 3, pp. 25652573, 2010.

[27] S. M. Mousavi, H. R. Rabiee, M. Moshref, and A. Dabirmoghaddam, "Mobisim: A framework for simulation of mobility models in mobile ad-hoc networks," in Third IEEE International Conference on Wireless and Mobile Computing, Networking and Communications (WiMob), 2007, pp. 82-82.

[28] N. Aschenbruck, R. Ernst, E. Gerhards-Padilla, and M. Schwamborn, "Bonnmotion: A mobility scenario generation and analysis tool," in Proceedings of the 3rd International ICST Conference on Simulation Tools and Techniques, ser. SIMUTools '10. Brussels, BEL: ICST (Institute for Computer Sciences, Social-Informatics and Telecommunications Engineering), 2010. [Online]. Available: https://doi.org/10.4108/ICST.SIMUTOOLS2010.8684

[29] R. R. F. Lopes, A. Viidanoja, M. Lhotellier, A. Diefenbach, N. Jansen, and T. Ginzler, "A queuing mechanism for delivering QoS-constrained web services in tactical networks," in International Conference on Military Communications and Information Systems (ICMCIS), Warsaw, Poland, 2018, pp. 1-8.

[30] N. R. Laboratory and P. E. A. N. R. Group, "MGEN user's and reference guide version 5.0." [Online]. Available: https://github.com/ USNavalResearchLaboratory/mgen/blob/master/doc/mgen.pdf

[31] A. Botta, A. Dainotti, and A. Pescapé, "A tool for the generation of realistic network workload for emerging networking scenarios," Computer Networks, vol. 56, no. 15, pp. 3531 - 3547, 2012.

[32] A. Botta, A. Dainotti, and A. Pescapé, "Do you trust your softwarebased traffic generator?" IEEE Communications Magazine, vol. 48, no. 9 , pp. $158-165,2010$.

[33] B. Liang and Z. J. Haas, "Predictive distance-based mobility management for pcs networks," in IEEE INFOCOM'99. Conference on Computer Communications. Proceedings. Eighteenth Annual Joint Conference of the IEEE Computer and Communications Societies. The Future is Now (Cat. No. 99CH36320), vol. 3, 1999, pp. 1377-1384.

[34] E. SMG, "Universal mobile telecommunications system (umts); selection procedures for the choice of radio transmission technologies of the umts," ETSI Document TR, vol. 101, p. 112, 1997.

[35] D. B. Johnson and D. A. Maltz, "Dynamic source routing in ad hoc wireless networks," in Mobile computing. Springer, 1996, pp. 153-181.

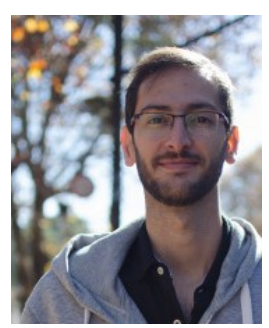

Paulo H. L. Rettore is a scientist at Fraunhofer FKIE in Bonn, Germany. He received his BSc. and MSc. degrees in Computer Science in 2009 and 2012, respectively. He got his Ph.D. degree in Computer Science at Federal University of Minas Gerais (UFMG) in 2019. His research interests include Computer Networks, Distributed Systems, Tactical Networks, Ubiquitous Computing, Internet of Things, Intelligent Transportation Systems and Smart Mobility.

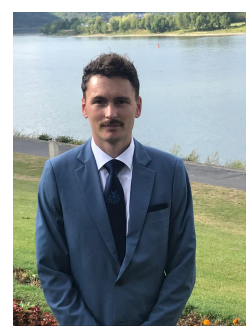

Johannes Loevenich is currently pursuing his masters in Computer Science at University of Bonn, Germany. $\mathrm{He}$ is working as a student assistant in Communication Systems Department (KOM) at Fraunhofer FKIE in Bonn, Germany. $\mathrm{He}$ received Bachelors degree in Computer Science at 2017 from Rheinische Friedrich-WilhelmsUniversität Bonn. One year later he also received a Bachelors degree in Mathematics from the same university. His research interests include Computer Systems, Distributed Systems, Tactical Networks, Data Science and Game Theory.

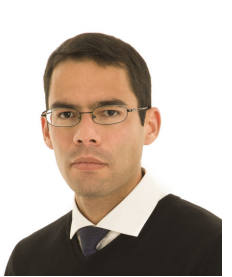

Roberto Rigolin F. Lopes (S'09, M'13) is a scientist at Fraunhofer FKIE in Bonn, Germany. Sitting with the Communication Systems Department (KOM), he has been attacking problems in Computer Networks and Distributed Systems with particular interest in the performance bounds of tactical systems over ever-changing communication scenarios. His education includes B.Sc., M.Sc. and Ph.D. degrees in Computer Science from three universities in Brazil (UFMT, UFSCar and USP). He also visited universities in the Netherlands (UTwente) and Norway (NTNU). But he rebuilt his own education following curiosity freely and reading books on Physics, Mathematics and Philosophy.

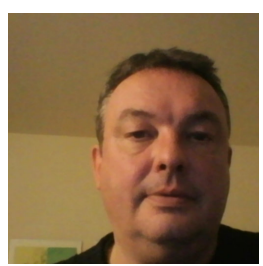

Peter Sevenich is the head of the Robust Heterogeneous Network group at the Communications Systems Department at Fraunhofer FKIE in Bonn, Germany. Peter got his degree in Physics from University of Bonn, Germany. He has participated in the conception and execution of several national/international projects developing military communications. His research interests include IP routing in Tactical Networks, Service-Oriented Architectures, Software Defined Networks and Software Defined Radios. 